\title{
V. Der Strafvollzug im Übergang von der Justiz- zur Innenverwaltung (1948-1952)
}

\section{Vorboten der Wende im Strafvollzugswesen 1948}

Wie in den anderen Aufgabengebieten des Justizwesens, kündigten sich 1948 auch im Strafvollzug einschneidende, auf neue sowjetische Vorgaben zurückgehende Veränderungen an, die dessen Unterstellungsverhältnis und dessen Reformorientierung betrafen. Infolge von SMAD-Befehl Nr. 201 vom 16. August 1947 wurde die Zuständigkeit der Justizverwaltung für den Strafvollzug von der Innenverwaltung in Frage gestellt. Denn die nach Ausführungsbestimmung Nr. 3 des Befehls in Untersuchungshaft zu nehmenden Hauptverbrecher mußten in Einzelhaft gehalten und in Polizeigefängnisse - und nicht, wie in Deutschland üblich, in Gerichtsgefängnisse - eingewiesen werden ${ }^{1}$. Polizeiangestellte wurden mit der Bewachung dieser Untersuchungshäftlinge auch in einzelnen Strafanstalten betraut, die auf Antrag gemäß Befehl Nr. 201 Festgenommene unterzubringen hatten². Kompetenzüberschneidungen und Reibungsflächen zwischen Justiz und Polizei waren daher unvermeidlich. Erheblich größere Probleme traten jedoch auf, als die Innenministerkonferenz am 29./30. November 1947 - an der neben den Innenministern der Länder und der Spitze der DVdI auch Ulbricht, Plenikowski und Karl Maron teilnahmen - beschloß, daß die „Strafvollstreckung“ gemäß Befehl Nr. 201 „den Polizeiorganen der Länder [...] unter der Kontrolle der Deutschen Verwaltung für Inneres" übertragen werden sollte. Als Einrichtungen für den Strafvollzug waren Lager mit Arbeitsmöglichkeiten in Bergwerken, Mooren und Steinbrüchen vorgesehen ${ }^{3}$. Dieser Versuch, die sowjetischen Bestimmungen über die Untersuchungshaft auch auf den Strafvollzug auszudehnen, traf jedoch auf den Widerstand der DJV. Melsheimer wandte sich am 9. Februar 1948 in der DVdI aus Gründen der Außenwirkung gegen diese Entscheidung: „Dadurch könne in der Westzone und im Ausland böswilligen Unterstellungen Raum gegeben werden, als werde diesen Verurteilten gegenüber nicht Gerechtigkeit geübt und als seien sie einer durch die Justiz nicht kontrollierbaren Verfolgung ausgesetzt.“ Mielke hingegen verteidigte den Polizeivollzug mit der etwas fadenscheinigen Begründung,

1 Ausführungsbestimmung Nr. 3 zum Befehl Nr. 201 vom 16. 8. 1947, Ziffer 7, 8, in: ZVOBl. 1947, S. 188f.; DJV: Erlaß zur Durchführung des Befehls Nr. 201 der SMAD vom 16. 8. 1947, Ziffer III.3, ebenda, S. 192; DVdI: Ausführungsbestimmungen über die Regelung der Feststellung und Registrierung von Hauptverbrechern, Verbrechern und Minderbelasteten, $₫ 5$, Abs. 4, BAB, DO1/7, Nr. 69, Bl. 34.

2 Chef der DJV an Landesregierungen/Justizministerium, 6. 2. 1948, BAB, DP1 HAS III/150, Bl. 36. Die Rundverfügung ist von Melsheimer und Mielke unterzeichnet.

3 Protokoll der Innenminister-Konferenz am 29./30. 11. 1947 in Schierke, in: Rößler, Entnazifizierungspolitik, S. 215. 
daß man die Verurteilten auf diese Weise „besser zu besonderen Wiedergutmachungsarbeiten heranziehen" könne. Eine Entscheidung fiel damals nicht; DVdI-Präsident Reschke wollte sich zuvor nochmals mit den „Referenten seines Amtes" und „eventuell“ auch mit Karlshorst beraten". Gentz bewog bei dieser Gelegenheit Reschke dazu, sich bei der Besatzungsmacht für die Freigabe der Strafanstalt Brandenburg-Görden für die nach Befehl Nr. 201 Verurteilten einzusetzen. Melsheimer wandte sich schriftlich an die SMAD-Rechtsabteilung, die den Antrag zur „Einrichtung besonderer Strafanstalten“ - Brandenburg-Görden wurde nicht explizit genannt - unterstützte ${ }^{5}$. Auch die DVdI, die Anfang März einen „Strukturplan für die Errichtung und den Betrieb von Häftlingslagern gemäß Befehl Nr. 201"6 ausarbeitete und der SMAD zur Genehmigung unterbreitete, wollte nun neben einzelnen Arbeitslagern in der SBZ eine „Stammanstalt in Brandenburg/Havel“ einrichten. Nachdem die Militärverwaltung einen Teil der Strafanstalt Brandenburg-Görden an den Chef der Landespolizeibehörde in Potsdam, Richard Staimer, durch die Militärverwaltung im März übergeben hatte ${ }^{7}$, brachte eine Mitteilung Jakupows vom 7. Juni endgültige Klarheit. Er informierte über einen Beschluß der SMAD, „den Strafvollzug an den aus Befehl 201 Verurteilten den Organen der Inneren Verwaltung zu übertragen" und diese in BrandenburgGörden zusammenzuziehen. Auf die erneut vorgebrachten Einwände, daß dadurch „ein falsches Bild entstehe, welches zu Propagandazwecken ausgenutzt werden wird, erwiderte Oberstleutnant Jakupow, daß eine evtl. propagandistische Wirkung bei der Lösung dieser Frage nicht maßgebend gewesen sei"8. Für die DJV bedeutete diese Entscheidung, die nicht in der SMAD-Rechtsabteilung gefallen war, eine erste Niederlage in der Auseinandersetzung um die Verfügungsgewalt über den Strafvollzug. Kurt Fischer, der am 3. Juli 1948 an Stelle von Reschke zum DVdI-Präsidenten ernannt worden war, machte auf der Innenministerkonferenz am 9./10. Juli deutlich, daß dies für ihn nur einen Etappensieg darstellte: „Die Justiz“, so Fischer, „müßte zum Gegenstand eingehender Untersuchung gemacht werden. Eine gründliche Justizreform ist dringend. Auf jeden Fall sollte der Strafvollzug dem Innenministerium (Polizei) übergeben werden." ${ }^{9}$ Die sowjetischkommunistische Prägung Fischers, der neben Ulbricht als „der einflußreichste, geschickteste und energischste Repräsentant des russischen Kurses" galt ${ }^{10}$, ist wohl auch für seine Bestrebungen auf diesem Gebiet verantwortlich zu machen.

Eine vollständige Übertragung der Geschäfte des Strafvollzugs auf die Innenverwaltung - was eine Anpassung an die Verhältnisse in der Sowjetunion bedeutet hätte -, kam damals für die Besatzungsmacht jedoch noch nicht in Frage. Auch die

4 Vermerk von Gentz, 17. 2. 1948, BAB, DP1 VA Nr. 6204, Bl. 108. Auch Ulbricht wurde von dieser Unterredung unterrichtet: siehe Plenikowski an Ulbricht, 12.2. 1948, SAPMO, NY 4182/1197, Bl. 232.

5 Chef der DJV an SMAD-Rechtsabteilung, 17. 2. 1948, BAB, DP1 VA Nr. 6204, Bl. 110; Äußerung Jakupows in: Bericht über die Besprechung bei der Rechtsabteilung in Karlshorst, 5. 3. 1948, BAB, DP1 VA Nr. 11, Bl. 131.

6 Strukturplan in Abschrift vom 11. 3. 1948, SAPMO, NY 4182/1197, Bl. $228 \mathrm{f}$.

7 Dies teilte Plenikowski Ulbricht am 9.3. 1948 mit, ebenda, Bl. 227.

8 Vermerk über Unterredung mit Jakupow, 7. 6. 1948, BAB, DP1 VA Nr. 11, Bl. 141.

9 Referat Fischers auf der Tagung der Innenminister am 9./10. 7. 1948, SAPMO, DY 30 IV 2/13/109, Bl. 326-330, hier 330. Vgl. Müller, Parteiministerien, S. 389.

10 So der Telegraf am 7. 11. 1948, zit. nach Richter/Schmeitzner, Einer von beiden, S. 77. 
Entscheidung, den Strafvollzug an den nach Befehl Nr. 201 Verurteilten der Polizei zu übertragen, wurde - aus bisher nicht bekannten Gründen - rückgängig gemacht ${ }^{11}$. Denn am 27. September übergab die Polizei auf Weisung der brandenburgischen SMA die für die Zusammenlegung der „201er“ vorgesehene Strafanstalt Brandenburg-Görden dem Strafvollzugsamt des Potsdamer Justizministeri$u s^{12}$. Nachdem am 27. Oktober Nikolajew mitgeteilt hatte, daß über den vorgesehenen Verwendungszweck der Anstalt endgültig im Sinne der DJV entschieden worden sei ${ }^{13}$, trat diese in Verhandlungen mit allen beteiligten Instanzen, um den Betrieb möglichst bald aufnehmen zu können ${ }^{14}$. Die Strafanstalt wurde zwar im Februar 1949 direkt der DJV unterstellt; aufgrund von Renovierungsarbeiten konnten jedoch erst im Mai 1949300 Gefangene übernommen werden ${ }^{15}$.

Die DJV registrierte 1948/49 zudem deutliche sowjetische Akzentverschiebungen bei den Themen Gefangenenarbeit und Sicherheit der Strafanstalten. So machte Karassjow am 31. August 1948 Melsheimer darauf aufmerksam, daß die ca. 7000 Strafgefangenen in der SBZ „ein großes Reservoir an Arbeitskraft [seien], welches zur Erfüllung des Planes für 1948 und des Zweijahresplanes herangezogen werden müsse" 16 . In der nächsten DJV-Dienstbesprechung am 15. September nannte Melsheimer daraufhin "die Nutzbarmachung der Gefangenen für den Zweijahresplan" neben der "Frage der Entweichungen" als eine der beiden von der SMAD-Rechtsabteilung als „vordringlich“ bezeichneten Aufgaben des Strafvollzugs ${ }^{17}$. Um den sowjetischen Wünschen gerecht zu werden, rückte die DJV die Gefangenenarbeit zunehmend in den Vordergrund ${ }^{18}$. Diese avancierte vom Mittel des Strafvollzugs zu dessen Hauptaufgabe, so daß auch bei dessen Zielsetzung die Prioritäten verschoben wurden: Stand unmittelbar nach 1945 die Resozialisierung der Gefangenen im Mittelpunkt, ging es nun primär darum, die ökonomischen Vorgaben der Wirtschaftsplaner zu erfüllen ${ }^{19}$. Freilich hatte für Gentz "die Nutzbarmachung des Arbeitspotentials der Strafanstalten“ nicht nur eine ökonomische, sondern auch eine erzieherische Bedeutung: „Pädagogisch gesehen muß die Mitarbeit am Zweijahresplan zur zentralen Triebfeder für die Resozialisierung der Gefangenen gemacht werden; ihnen muß die Idee des Zweijahresplanes zum Motiv des persönlichen Arbeitseinsatzes werden; sie müssen zur Mitverantwortung herangezogen werden und damit das Bewußtsein gewinnen, daß sie Mitgestalter des Arbeitsplanes und damit ihres eigenen Geschickes sind. [...] Die Gefangenen dürfen nicht nur die Arbeitsinstrumente zur Erzielung von ökono-

11 Dies wird von Meyer-Seitz, Verfolgung von NS-Straftaten, S. $326 \mathrm{f}$., übersehen.

12 MdJ Brandenburg an Chef der DJV, 18. 10. 1948, BAB, DO1/11 Nr. 1589, Bl. 1; vgl. auch Vermerk Gentz, o.D., ebenda, Bl. 3, über eine entsprechende Mitteilung der DVdI vom 16. 10. 1948.

13 Vermerk Walters, 27. 10. 1948, ebenda, Bl. 2.

i4 Siehe dazu Vermerke von Gentz vom 2. und 22.11. 1948 über Besprechungen am 2. und 16.11. 1948 , BAB, DO1/11, Nr. 1589, Bl. 4-6, 9-15.

15 Halbjahresbericht Strafvollzug, 13. 7. 1949, BAB, DP1 HAS III/145.

16 Vermerk über eine Unterredung zwischen Karassjow und Melsheimer, 31. 8. 1948, BAB, DP1 VA Nr. 11, Bl. 153.

17 Vermerk über die Dienstbesprechung vom 15. 9. 1948, BAB, DP1 VA Nr. 7354.

18 Vgl. u.a. die Disposition für den Vortrag Fechners in Karlshorst am 9. 2. 1949, BAB, DP1 VA Nr. 3, Bl. 207-216, hier 216; Arbeitsplan für die Abteilung II/4 [für das 1. Halbjahr 1949], BAB, DP1 VA Nr. 6214.

is Vgl. auch Oleschinski, Abteilung Strafvollzug, S. 88, die jedoch nicht zeitlich differenziert. 
mischen Leistungen sein, sondern müssen dazu gebracht werden, im Rahmen eines Arbeitskollektives an der Arbeitsplanung und Leistungssteigerung selbsttätig Anteil zu nehmen. " 20 Gentz hielt also auch unter den geänderten Vorgaben an seiner Konzeption eines erzieherischen Strafvollzugs fest.

Vorerst blieb die Einbeziehung der Gefangenenarbeit in den Zweijahresplan jedoch mehr Wunsch als Realität. Im November 1948 vereinbarten die DJV und die Hauptverwaltung Arbeit und Sozialfürsorge in der DWK, unter der Federführung der Wirtschaftsplanungsstellen Länderkommissionen zu bilden, „die die Möglichkeit des Einsatzes von Strafgefangenen in den Strafanstalten selbst oder in Kolonnenarbeit in den Betrieben prüfen sollten"21. Die Hauptverwaltung Arbeit und Sozialfürsorge (HVAS) und die DJV erkannten im folgenden Jahr, daß die Ergebnisse dieser Maßnahmen insgesamt dürftig waren. Im März 1949 gaben Vertreter der beiden Verwaltungen zu, „daß der Zeitpunkt für eine umfassende Einplanung der arbeitsfähigen Insassen von Strafanstalten in den Zweijahrplan noch nicht geeignet" sei22. Die Berichte der DJV bestätigten, daß weniger als 50 Prozent aller Strafgefangenen im produktiven Arbeitseinsatz waren ${ }^{23}$. Beide Verwaltungen machten vor allem die schlechte Arbeitsmarktlage dafür verantwortlich. Die HVAS hob zudem die mangelhafte Ausstattung der Strafanstalten mit Rohstoffen und Maschinen hervor ${ }^{24}$. Die DJV wiederum sah 1948 eine wesentliche Ursache darin, daß die Gefangenenarbeit nicht zentral geplant, sondern den einzelnen Strafanstaltsdirektoren überlassen wurde, die mit dieser Aufgabe überfordert waren ${ }^{25}$, und kritisierte im Jahr darauf den mangelnden Einsatz der einzelnen Wirtschaftsplanungsstellen und Landesarbeitsämter sowie die dezentrale Organisation der Arbeitsbeschaffung. Schließlich machte sie auch die regionalen Militärverwaltungen für eine Erschwerung der Gefangenenarbeit verantwortlich, die in Mecklenburg und Thüringen die Beschäftigung von Gefangenen bei Außenarbeiten eingeschränkt hatten, um die Zahl der Entweichungen zu reduzieren ${ }^{26}$. Da die sowjetische Besatzungsmacht ab 1948 ebensoviel Wert auf Sicherheit wie auf Gefangenenarbeit legte, kam es hier anscheinend zu Zielkonflikten, die eher zugunsten der Sicherheit der Verwahrung als der möglichst umfassenden Arbeitsbeschaffung entschieden wurden.

Die Sicherheit der Anstalten zu erhöhen und Ausbrüche zu verhindern, war das zweite wesentliche sowjetische Interesse am Strafvollzug in den Jahren 1948/49. Die SMAD-Rechtsabteilung forderte nun vermehrt Berichte über den Strafvoll-

20 Chef der DJV an Landesregierungen/Justizministerium, 27.12. 1948, BAB, DP1 VA Nr. 839, Bl. 42-44, hier $42 \mathrm{f}$. Gentz hatte die Rundverfügung entworfen.

21 Vgl. dazu Rundverfügung der DJV, 18. 8. 1949, und HVAS an SMAD, Abt. „Die Arbeitskraft“, 6. 8. 1949, BAB, DQ2, Nr. 2022. Den Hinweis auf diese Archivalien verdanke ich Dr. Dierk Hoffmann.

22 HVAS an SMAD, Abt. „Die Arbeitskraft“, 6. 8. 1949, ebenda.

23 Nach dem Bericht zur Lage des Strafvollzuges in der Sowjetischen Besatzungszone vom Mai 1949 (BAB, DP1 HAS III/114) waren nur 50\% der Strafgefangenen überhaupt im Arbeitseinsatz. Im „produktiven Arbeitseinsatz“ im engeren Sinne waren laut Halbjahresbericht vom 13. 7. 1949 nur 35 bis $40 \%$, BAB, DP1 HAS III/145.

24 HVAS an SMAD, Abt. „Die Arbeitskraft“, 16. 8. 1949, BAB, DQ2 Nr. 2022.

25 Bericht von Gentz über die Dienstreise nach Untermaßfeld, Eisenach, Gräfentonna, Ichtershausen, Naumburg und Coswig vom 10.-13. 8. 1948, BAB, DP1 HAS III/150, Bl. 137-141, hier 140.

26 Zur Lage des Strafvollzuges in der Sowjetischen Besatzungszone, Mai 1949, BAB, DP1 HAS III/ 114; Halbjahresbericht Strafvolizug, 13. 7. 1949, BAB, DP1 HAS III/145. 
zug und insbesondere die Anzahl der Fluchtfälle27. Die daraufhin angefertigten Ausarbeitungen zeigen indes, daß die Entweichungszahlen insgesamt eine rückläufige Tendenz aufwiesen. Waren in den Monaten Januar bis Oktober 1947 insgesamt 1918 Gefangene ausgebrochen, so waren es im gleichen Zeitraum des Jahres 1948 nur noch 162628; im ersten Halbjahr 1949 entkamen insgesamt $426 \mathrm{Ge}$ fangene, während im zweiten Halbjahr 1948 die Zahl bei 953 gelegen hatte. Auch die monatlichen Entweichungsziffern sanken von August 1948 bis Juni 1949 sowohl in absoluten als auch in Prozentzahlen kontinuierlich ${ }^{29}$. Vor allem die bessere Bewaffnung des Personals, dessen eingehende Unterrichtung über seine Dienstpflichten und die energische Verfolgung von Nachlässigkeiten bei der Gefangenenbewachung trugen zu diesen Erfolgen bei ${ }^{30}$. Dennoch bezeichnete die DJV, um weiteren sowjetischen Beanstandungen und dem Vorwurf der Untätigkeit zu entgehen, am 22. Dezember 1948 gegenüber den Landesjustizministerien die Zahl der Ausbrüche als "noch immer sehr erheblich" und übersandte gleichzeitig ein Merkblatt für die Strafanstalten mit einer Reihe von Richtlinien, „bei deren sorgfältiger Beachtung viele Entweichungen verhindert werden können " 31 .

Die DJV erwog außerdem seit März 1948 - möglicherweise auf sowjetische Anregung hin - „die Selbstbefreiung Gefangener unter eine besondere Strafandrohung zu stellen"32. Gentz traf damit auf der Länderkonferenz am 12./13. März auf wenig Gegenliebe: Abgesehen vom brandenburgischen Justizminister Stargardt, sprachen sich alle Ländervertreter vor allem aus Zweckmäßigkeitsgründen dagegen aus. Unterstützt wurde Gentz nur von Schiffer und - im Namen der SMADRechtsabteilung - von Major Nikolajew. Ein „endgültiger Beschluß“ sollte jedoch auch nach dessen Bekundungen nicht gefaßt werden ${ }^{33}$. Im Herbst 1948, als das verstärkte sowjetische Interesse an der Sicherheit der Anstalten unübersehbar geworden war, arbeitete die Strafvollzugsabteilung einen Gesetzesentwurf aus, „nach welchem Gefangene, die entweichen, mit Zusatzgefängnisstrafe bis zu 2 Jahren bestraft werden können "34. Obwohl die SMAD-Rechtsabteilung dem am 6. Dezember eingereichten Entwurf ${ }^{35}$ inhaltlich zustimmte, verzögerte sich dessen Umsetzung. Zunächst lehnte sie eine Verordnung ab, da sie angeblich nicht ermächtigt sei, „eine Strafbestimmung enthaltende VO von sich aus zu bestätigen“, und bat um einen Befehlsentwurf ${ }^{36}$, den die DJV am 8. Januar 1949 ein-

27 Vgl. die Berichtsaufträge Jakupows vom 20.8. 1948 und Karassjows vom 11. 12. 1948 in den Berichten vom 25. 9. 1948 und 21. 12. 1948, BAB, DP1 VA Nr. 7855, DP1 HAS III/145.

28 Ebenda.

29 Halbjahresbericht Strafvollzug, 13. 7. 1949, BAB, DP1 HAS III/145: Im August 1948 waren von 16618 Gefangenen 208 entflohen; im Juni 1949 waren von 2185663 ausgebrochen.

30 DJV an SMAD-Rechtsabteilung, 21. 12. 1948, BAB, DP1 HAS III/145.

31 Chef der DJV an Landesregierungen/Justizministerium, 22.12. 1948, BAB, DP1 HAS III/150, Bl. 164. Das Merkblatt war dem Aktenexemplar der Rundverfügung nicht beigefügt; vgl. Oleschinski, Abteilung Strafvollzug, S. 86.

32 Chef der DJV an SMAD-Rechtsabteilung, 1. 3. 1948, BAB, DP1 VA Nr. 6958, BI. 119-121.

33 Protokoll der Länderkonferenz vom 12. 3. 1948 [unvollständig], ebenda, Bl. 139-144, hier 142145. Vgl. auch den Bericht über die Länderkonferenz von einem DVdI-Vertreter vom 16. 3. 1948, BStU, MfS AS 230/66, Bl. 49-55, hier 52-54.

34 DJV an SMAD-Rechtsabteilung, 25. 9. 1948, BAB, DP1 VA Nr. 7855.

35 Siehe DJV an SMAD-Rechtsabteilung, 21. 12. 1948, BAB, DP1 HAS III/145.

36 Vermerk über eine Unterredung von Dozenko, Gentz, Meyer, Lange am 20. 12. 1948, BAB, DP1 VA Nr. 11, Bl. 156. 
reichte ${ }^{37}$. Nun wurde jedoch in Karlshorst entschieden, die Bestrafung von Gefangenenentweichungen per Landesgesetz zu regeln. Obwohl die Rechtsabteilung am 4. März 1949 versicherte, sie werde „von sich aus auf die zuständigen Stellen der SMA in den Ländern einwirken, um zu erreichen, daß die Gesetze beschleunigt und einheitlich erlassen werden " 38 , blieb es - aus ungeklärten Gründen - bei dieser Ankündigung, und ein Rechtsakt zur Bestrafung von Gefangenentweichungen wurde in keinem der fünf Länder verabschiedet ${ }^{39}$.

\section{Zwischen Strafvollzugsreform und Übernahmeversuchen durch die Innenverwaltung: Der Strafvollzug im Jahre 1949}

Die weitere Entwicklung im letzten Jahr der SBZ war geprägt von den Bestrebungen der DJV, den Strafvollzug bei der Justiz zu behalten, um das Reformprogramm in eigener Regie fortführen zu können. So bezeichnete Fechner am 9. Februar 1949 in Karlshorst den Strafvollzug als "noch völlig ungenügend“. Als vordringliche Aufgaben nannte er neben der Einbeziehung der Gefangenen in die Arbeiten des Zweijahresplanes deren Differenzierung, die Schaffung von Gefangenen-Arbeitskollektiven, die Bildung von Strafvollzugsausschüssen und den Ausbau des Bewährungseinsatzes. Dies waren Vorhaben, die Gentz seit längerer Zeit verfolgte, die nun jedoch durch einen Ausbau der Strafvollzugsabteilung und durch eine stärkere Zentralisierung realisiert werden sollten ${ }^{40}$. Fechner begründete das stärkere Engagement der DJV gegenüber der Rechtsabteilung mit der mangelnden Initiative der Länder und kündigte an, die Strafvollzugsabteilung „zu einer Abteilung für Strafvollzug und Gefängnisbetreuung mit dem Ziele straffer, zentraler Leitung und Überwachung des Gefängniswesens“ zu erweitern. Dazu wollte er innerhalb der Abteilung fünf Referate einrichten, die jeweils für den Strafvollzug im allgemeinen, für die Gefangenenarbeit, für Schulung und Soziales, für technische Angelegenheiten und für die Strafanstalt Brandenburg-Görden zuständig sein sollten ${ }^{41}$. Bei der SMAD-Rechtsabteilung traf er damit auf Verständnis, und Korobow erklärte sich am 13. April mit der Stellenvermehrung der Abteilung „um ein Arbeits- und Schulungsreferat" einverstanden ${ }^{42}$. Angesichts der Bestrebungen der SMAD-Innenverwaltung seit Mai 1949, die Zuständigkeit der Justizverwaltung für den Strafvollzug in Frage zu stellen, rückten in der SMAD-Rechtsabteilung und in der DJV Strafvollzugsangelegenheiten mehr in den Mittelpunkt: In der DJV fanden vermehrt Dienstbesprechungen zu dieser Thematik statt, von denen eine sogar auf Veranlassung der SMAD-Rechtsabteilung abgehalten wurde ${ }^{43}$. Fechner wies bei dieser Gelegenheit Vizepräsident

37 Chef der DJV an SMAD-Rechtsabteilung, 8. 1. 1949, BAB, DP1 HAS III/145.

38 Vermerk über eine Unterredung mit Nikolajew und Korobow am 4.3. 1949, BAB, DP1 VA Nr. 11, Bl. 161.

39 Dies ergab eine Durchsicht der Gesetz- und Verordnungsblätter der Länder.

40 Disposition für den Vortrag Fechners in Karlshorst am 9. 2. 1949, BAB, DP1 VA Nr. 3, Bl. $215 \mathrm{f}$.

41 Chef der DJV an SMAD-Rechtsabteilung, 17. 2. 1949, BAB, DP1 HAS III/145.

42 Vermerk über eine Unterredung Korobows mit Gentz am 13. 4. 1949, 14. 4. 1949, BAB, DP1 HAS III/114.

43 Chef der DJV an Ljulka, 29. 7. 1949, BAB, DP1 VA Nr. 3, Bl. 300. 
Brandt scharf wegen Vernachlässigung des ihm zugewiesenen Aufgabengebiets zurecht und betonte sein persönliches Engagement für den Strafvollzug, dem „bis zu seinem Dienstantritt bedauerlicherweise nicht die gebührende Beachtung geschenkt worden" sei ${ }^{44}$.

Angesichts des persönlichen Einsatzes von Gentz und Poelchau, der zum 31. März 1949 aus der DJV ausgeschieden war ${ }^{45}$, traf diese Behauptung in keiner Weise zu. Die widrigen Umstände der unmittelbaren Nachkriegszeit hatten jedoch die Strafvollzugsreform stark behindert. Ein von sowjetischer Seite angeforderter Bericht „Zur Lage des Strafvollzugs in der Sowjetischen Besatzungszone“ vom Mai 1949 zeigt, daß sich diese Bedingungen trotz aller Anstrengungen auch 1949 noch nicht grundlegend gewandelt hatten. Darin kamen unter anderem die Überfüllung der Anstalten, die schlechte Ausstattung der Gefangenen mit Kleidung und Bettwäsche, die katastrophalen Ernährungslage, die mangelnden Arbeitsmöglichkeiten, die ungenügende Ausbildung des Anstaltspersonals und das niedrige kulturellen Niveau der Anstalten zur Sprache. Die meisten Vorschläge von Gentz zur Behebung dieser Mängel krankten daran, daß sie essentiell von der Kooperation anderer Einrichtungen auf Landes- und Zonenebene abhingen. Gleichzeitig nutzte er die Gelegenheit, um darauf hinzuweisen, daß die DJV jeweils einen Referenten für Arbeitsplanung und Schulung benötige, um die Gefangenenarbeit effektiv organisieren und eine Schulungsstätte errichten und betreuen zu können46. Die Bemühungen um Erweiterung des Stellenplans für die Strafvollzugsabteilung hielten bis zum September an ${ }^{47}$; bemerkenswert dabei ist, daß die SMAD-Rechtsabteilung damit - wie auch mit der geplanten Ausgliederung der Abteilung Strafvollzug aus der Hauptabteilung „Kontrolle“ und deren Aufwertung zu einer eigenen Hauptabteilung - grundsätzlich einverstanden war ${ }^{48}$, aber anscheinend nichts tat, um die Realisierung dieser Vorhaben zu beschleunigen. Erst im Zuge des Übergangs der DJV zum MdJ erfolgte die Bildung einer selbständigen Hauptabteilung Strafvollzug und eine Stellenvermehrung: Dem genehmigten Strukturplan des MdJ vom 26. November 1949 zufolge wies die Hauptabteilung drei Hauptreferenten- und drei Oberreferentenstellen auf, von denen jedoch nur jeweils eine besetzt war ${ }^{49}$.

44 Protokoll über die Dienstbesprechung bei Fechner am 18. 7.1949 mit dem Thema: „Der Strafvollzug und seine vordringlichen Aufgaben", ebenda, Bl. 301-303. Darin sind auch die anderen Besprechungen zur selben Thematik vom 11.5. und 13.7.1949 erwähnt.

45 Siehe Verzeichnis der seit 1945 ausgeschiedenen Angehörigen der DJV, BAB, DP1 VA Nr. 1009, Bl. 70. Poelchau schied aus, weil er sich „von der Übernahme des Strafvollzuges aus der Justiz in die Polizei keine Möglichkeit eines Erziehungsstrafvollzugs versprach": siehe Poelchau, Ordnung, S. 110.

46 Der Berichtsauftrag Korobows in: Vermerk Langes, 5. 5. 1949, BAB, DP1 HAS III/114; der Bericht zur Lage des Strafvollzugs in der Sowjetischen Besatzungszone, Mai 1949, ebenda. Vgl. auch den Halbjahresbericht Strafvollzug, 13. 7. 1949, BAB, DP1 HAS III/145.

47 Am 14. 7. 1948 wurde bei der DWK die Erweiterung der Abteilung Strafvollzug unter anderem um 2 Haupt- und 2 Oberreferenten beantragt; am 22. September wurde beantragt, eine der drei Hauptreferentenstellen in eine Abteilungsleiterstelle umzuwandeln, BAB, DP1 VA Nr. 7855.

48 Vermerk über eine Besprechung zwischen Dozenko, Korobow und Brandt am 2. 9. 1949, BAB, DP1 HAS III/145.

49 Strukturplan des MdJ, 26. 11. 1949, BAB, DP1 VA Nr. 1, Bl. 342-347, hier 347. In Entwürfen des MdJ vom 6. und 25. 10. 1949 war unter dem Hauptabteilungsleiter noch ein Abteilungsleiter vorgesehen; nach einer Besprechung am 2. 11. mit dem Finanz- und dem Innenministerium wurde die Stelle gestrichen: BAB, DP1 VA Nr. 5577, Bl. 2-6, 24-27, $35 \mathrm{f}$. 
Verzögert wurden auch die Arbeiten an einer Strafvollzugsordnung. 1949 galt grundsätzlich noch die Strafvollzugsordnung aus dem Jahre 194050, die jedoch von allen nationalsozialistischen Bestimmungen gereinigt worden war ${ }^{51}$. Die SMAD-Rechtsabteilung hatte zwar im Dezember 1946 die Ausarbeitung einer neuen Strafvollzugsordnung unter Berücksichtigung von Kontrollratsdirektive Nr. 19 gefordert ${ }^{52}$; weder die Rechtsabteilung noch Gentz hatten dies jedoch forciert, wobei Gentz vermutlich einen Alleingang der SBZ vermeiden wollte und eine gesamtdeutsche Lösung anstrebte ${ }^{53}$. Am 18. Juni 1949 bat Dozenko - ganz im Einklang mit dem damals vorherrschenden Drang zur Vereinheitlichung und Zentralisierung der Justiz -, die Ausarbeitung einer neuen Vollzugsordnung zu beschleunigen. Mitte September 1949 reichte Brandt den Entwurf einer neuen Strafvollzugsordnung in Karlshorst ein, deren Durchsicht bis zum 25. September zugesichert wurde, damit sie, wie von der DJV geplant, am 1. Oktober in Kraft gesetzt werden könne ${ }^{54}$. Trotz dieser genau terminierten Zusage und des offensichtlichen Interesses der Rechtsabteilung an einer Strafvollzugsordnung blieb eine Antwort aus - möglicherweise ein Indiz dafür, daß der Entwurf einer anderen Stelle innerhalb der SMAD nicht behagte. Erst bei einer Unterredung am 29. Dezember teilte Korobow mit, daß "unter den veränderten Verhältnissen“ - also nach Gründung der DDR und der Bildung der SKK als Nachfolgeorganisation der SMAD - eine solche Genehmigung nicht mehr erforderlich und der Erlaß einer Strafvollzugsordnung „eine rein innere Angelegenheit der Deutschen Republik [sic]" sei ${ }^{55}$. Gentz plante auch den Erlaß eines Rahmengesetzes für den Strafvollzug, um der Vollzugsordnung "die rechtliche Grundlage zu geben“. Dies hielt Korobow „von seinem Standpunkt als Jurist“ zwar für richtig und erklärte sich „zu konsultativer Beratung" bereit; erforderlich sei eine sowjetische Mitwirkung jedoch nicht. Der SKK-Vertreter schob anscheinend die formell reduzierte $\mathrm{Zu}$ ständigkeit der sowjetischen Kontrollbehörde gegenüber der SMAD vor, um sich in der zwischen Innenverwaltung und Justizverwaltung kontroversen Frage des Strafvollzugs nicht festlegen zu müssen.

Mit dem um die Jahreswende 1949/50 ausgearbeiteten Strafvollzugsgesetz wollte Gentz sowohl die Strafvollzugsreform - der Entwurf enthielt Bestimmungen zur Ausbildung der Strafvollzugsangestellten, zur Gefangenenarbeit, zur "Kulturpflege" in den Anstalten und zur Schaffung von Strafvollzugsausschüssen - als auch den Verbleib des Strafvollzugs im Zuständigkeitsbereich des Justizministeriums gesetzlich verankern ${ }^{56}$. Am 6. Januar 1950 entschied Fechner jedoch, davon abzusehen, da ein solches Gesetz für eine Anweisung für den Strafvollzug

50 Vgl. dazu Möhler, Strafvollzug, S. 49 f.

51 So Gentz am 5. 5. 1949, zit. nach Oleschinski, Abteilung Strafvollzug, S. 87.

52 Vermerk über die Besprechung der Abteilung IV A der DJV in Berlin-Karlshorst am 18.12. 1946, BAB, DP1 VA Nr. 7855.

53 Vgl. Oleschinski, Abteilung Strafvollzug, S. 87.

${ }_{54}$ Vgl. ebenda, S. 88, und Vermerk über eine Besprechung mit Titow und Korobow am 13. 9. 1949, BAB, DP1 VA Nr. 6220.

s5 Vermerk über die Besprechung mit Korobow am 29. 12. 1949, 29. 12. 1949, ebenda; vgl. Oleschinski, Abteilung Strafvollzug, S. 88.

56 Entwurf des Strafvollzugsgesetzes, BAB, DP1 VA Nr. 7204, Bl. 5-8. 
nicht erforderlich sei57. Fechner war anscheinend nicht bereit, den offenen Widerspruch des Innenministeriums zu riskieren; außerdem hatte er sich im August 1949 bereits gegenüber Fischer und einem Vertreter der Besatzungsmacht auf eine andere Linie hinsichtlich des endgültigen Verbleibs des Strafvollzugs festgelegt ${ }^{58}$. Die Initiative von Gentz wurde somit durch taktische Überlegungen seines Ministers erfolgreich konterkariert. Damit teilte sie das Schicksal der Strafvollzugsordnung, die am 15. Februar von Korobow nochmals angemahnt, dann aber doch nicht mehr erlassen wurde ${ }^{59}$.

Die Bemühungen der Justizverwaltung zur Fortführung der Strafvollzugsreform in eigener Regie richteten sich nicht zuletzt gegen die Versuche der Innenverwaltung zur Übernahme des Strafvollzugs. Das Jahr 1949 läutete eine weitere Runde in der Auseinandersetzung zwischen DJV und DVdI ein. Ansatzpunkt war diesmal die von sowjetischer Seite monierte mangelhafte Sicherheit der Gefängnisse. Karassjow regte daher am 5. Mai gegenüber Brandt an, die Außenposten der Anstalten mit Polizisten zu besetzen, was dieser jedoch unter Verweis auf daraus resultierende Kompetenzstreitigkeiten zwischen Innen- und Außenbewachung zurückwies ${ }^{60}$. Dieser Vorschlag ging, wie Korobow tags darauf Gentz mitteilte, auf die SMAD-Innenverwaltung zurück. Die Rechtsabteilung erklärte sich prinzipiell einverstanden, ordnete aber an, ,jegliche Einmischung der Polizei in den inneren Dienstbetrieb der Anstalt, in die Erziehung der Gefangenen, in die Arbeit der Gefangenen " zu vermeiden. Auch Gentz erklärte sich zwar einverstanden, wollte aber die Kompetenzen der Polizei auf die nächtliche Außenkontrolle der Anstalten, die Übernahme von Gefangenentransporten und die Bewachung von größeren Gefangenenkolonnen bei Außenarbeiten beschränken. Korobow stimmte zu und betonte nochmals, „daß den Polizeikräften keine Einmischung in den Strafvollzug selbst gestattet werden solle“. Sowohl Gentz als auch Korobow waren offensichtlich redlich darum bemüht, die Auswirkungen dieser Initiative der SMAD-Innenverwaltung auf den Strafvollzug eng zu begrenzen. Korobow nahm sogar in Aussicht, daß nach Rücksprache mit der SMAD-Innenverwaltung eine gemeinsame Instruktion über die Aufgabenverteilung von Polizei und Justiz unter dem Vorsitz Fechners in der DJV ausgearbeitet werden sollte, „damit die Interessen der Justizverwaltung in jedem Sinne gewahrt würden“. Fechner sprach sich ebenfalls dafür aus und hob abschließend hervor, „daß er aus politischen Gründen es für richtig halte, daß der Strafvollzug nicht der Verwaltung des Innern unterstellt werde, sondern bei der Justiz verbleibe" 61.

Im Monat darauf wurden in Karlshorst offensichtlich noch sehr viel weitergehende Überlegungen angestellt. Denn in einer Dienstbesprechung am 23. Juni, an der neben Brandt, Scheele, Gentz und zwei seiner Mitarbeiter auch DVdI-Präsident Fischer teilnahm, gab Fechner bekannt, daß die SMAD die Strafvollzugsent-

57 Vermerk von Weiß über eine Besprechung bei Fechner am 6. 1. 1950, 7. 1. 1950, ebenda, Bl. 3. Diese Entscheidung teilte er am 10.1.1950 der SKK mit, die über den Entwurf des Strafvollzugsgesetzes informiert werden wollte, BAB, DP1 HAS III/145.

58 Siehe unten, S. 379.

59 Vermerk über die Rücksprache in Karlshorst am 15. 2. 1950 mit Herrn Korobow, ebenda.

60 Vermerk Brandts über Besprechung mit Karassjow am 5. 5. 1949, BAB, DP1 VA Nr. 6220.

61 Niederschrift über die Besprechung mit Korobow am 6. 5. 1949, BAB, DP1 HAS III/145. 
wicklung als äußerst unbefriedigend einschätze, und berichtete „über die erwogene Übernahme des Strafvollzugs in die Verwaltung des Innern, seinen einstweiligen Verbleib bei der Justizverwaltung und stellt $[\mathrm{e}]$ als seine vordringlichsten Aufgaben 1. die Arbeitsbeschaffung für die Gefangenen und 2. die Erziehung der Gefangenen in den Vordergrund, wobei er betont [e], daß der zu stark ausgeprägte humane Strafvollzug im Augenblick erst in zweiter Linie in Erscheinung zu treten habe". Wieder hatte es anscheinend sowohl innerhalb der SMAD als auch zwischen den beiden Zentralverwaltungspräsidenten Auseinandersetzungen um den Strafvollzug gegeben, die indes noch einmal zugunsten der Justizverwaltung ausgegangen waren. Die Justiz konnte nun jedoch nicht mehr mit ihrem Kurs so fortfahren, als wäre nichts geschehen. Im Gegenteil: Da Fechner bereit war, alles zu tun, „um den Verbleib des Strafvollzugs bei der Justiz zu rechtfertigen“, mußte er den Vorwürfen der Innenverwaltung den Wind aus den Segeln nehmen. Daher gab er sich vor dem DVdI-Präsidenten als Verfechter einer harten Linie: Die Humanisierung des Strafvollzugs werde nun dessen „vordringlichsten Aufgaben“ untergeordnet und die Sicherheit der Anstalten „durch eine gut funktionierende Bewachung bez. Erhöhung des Aufsichtspersonals" verstärkt ${ }^{62}$. Da die DVdI die Strafvollzugspraxis kritisch beobachtete und ihr aufgefallene Mängel an Karlshorst weitermeldete ${ }^{63}$, mußte Fechner diesen Worten Taten folgen lassen, wenn der Strafvollzug auch weiterhin bei der Justiz ressortieren sollte.

Die Innenverwaltung blieb hartnäckig. Ein weiterer Versuch, den Einfluß der Polizei auch auf den Strafvollzug auszudehnen, erfolgte über das Kleine Sekretariat des Politbüros. Ganz im Sinne der DVdI beschloß es am 12. August 1949 angesichts der angeblichen Unfähigkeit der Justizorgane, „die Flucht und die unzulässige Freilassung von Gefangenen zu verhindern", der SMAD vorzuschlagen, in jeder Strafanstalt als stellvertretenden Direktor einen Angestellten der Volkspolizei einzusetzen. Ohne dessen Gegenzeichnung sollten in Zukunft keine Untersuchungsgefangenen entlassen werden. Der Vorschlag der DJV, das Strafanstaltspersonal der Polizei gleichzustellen, war nur dann durchzuführen, „wenn die Gefängnisangestellten in die Polizei eingegliedert werden"64. Dieser Beschluß zeigt zum einen, daß die Innenverwaltung aufgrund ihrer engen Verbindung zur SEDFührung unter Ulbricht am längeren Hebel saß, und zum anderen, daß die Interessen der Justiz im Kleinen Sekretariat nicht wahrgenommen wurden. Fechner reagierte am 23. August mit einer ausführlichen Stellungnahme, die er offensichtlich auch der SMAD-Rechtsabteilung zukommen ließ65. Darin führte er zunächst

62 Niederschrift über die Dienstbesprechung über Strafvollzugsangelegenheiten vom 23.6. 1949, BAB, DP1 HAS III/150, Bl. 176-178. Vgl. dazu auch Fechners Äußerung von Ende Januar 1950: "Den Begriff ,humaner Strafvollzug' haben wir gestrichen, weil er zu verschieden ausgelegt wird.“ (in: Staatliche Kontrolle - Volkskontrolle, S. 138).

63 Die DVdI hatte beispielsweise der SMAD am 30. 3. 1949 Beanstandungen über den Strafvollzug an „201ern" und an einer "Wirtschaftsverbrecherin“ gemeldet, was Korobow veranlaßte, die DJV um Abstellung der Mängel zu bitten: siehe Vermerk über eine Unterredung Korobows mit Gentz am 13. 4. 1949, BAB, DP1 HAS III/114. Fischer bat die Landesbehörden der VP am 28. 6. $1949 \mathrm{um}$ Berichte über ungesetzliche Beurlaubungen, Entweichungen usw.: vgl. Pohl, Justiz in Brandenburg (Manuskript), S. 127.

64 Protokoll der Sitzung des Kleinen Sekretariats, 12. 8. 1949, SAPMO, DY 30 IV J 2/3/46.

65 Fechner an Zentralsekretariat der SED, Abt. Justiz, 23. 8. 1949, BAB, DP1 VA Nr. 262, Bl. 96; die Stellungnahme in deutscher Sprache und in russischer Übersetzung ebenda, Bl. 97-102, 142-147. 
an, daß die Entweichungen sowohl in absoluten Zahlen als auch in Prozentzahlen seit Sommer 1948 ständig bis auf einen Tiefststand von 0,2 Prozent gesunken seien. Auch die Polizei biete keine zuverlässige Garantie gegen Ausbrüche: Denn im ersten Quartal 1948 seien trotz ungleich besserer Bewachung aus den sächsischen Polizeihaftlagern prozentual zehnmal so viele Gefangene wie aus den Justizgefängnissen geflohen; außerdem erfolgten die meisten Entweichungen bei Außenarbeiten und bei von der Polizei durchgeführten Transporten. Zweitens mußte Fechner zufolge die Aufteilung der Leitungsbefugnisse auf zwei Direktoren aus unterschiedlichen Verwaltungen zwangsläufig zu Kompetenzgerangel führen, was dem Strafvollzug eher schade als nütze. Drittens wies er die Beschuldigung zurück, daß die Justizorgane nicht in der Lage seien, „die unzulässige Freilassung von Gefangenen zu verhindern“, und machte darauf aufmerksam, daß die Forderung nach Gegenzeichnung bei der Entlassung von Untersuchungsgefangenen der Strafprozeßordnung widerspreche; dafür sei allein der Untersuchungsrichter zuständig. Von der Änderung der noch in ganz Deutschland gültigen Strafprozeßordnung riet er aus deutschlandpolitischen Gründen ab, empfahl aber eine informelle Regelung, derzufolge der Richter einen Untersuchungsgefangenen erst nach Zustimmung der Volkspolizei entlassen solle. Viertens verteidigte er seinen Antrag auf Gleichstellung der Strafvollzugsangestellten mit den Volkspolizisten, da nur so "qualifizierte Kader für den Strafvollzug zu gewinnen“ seien. Ungeachtet dieser gewichtigen Argumente gegen den Sekretariatsbeschluß machte Fechner jedoch keine prinzipiellen, sondern lediglich taktische Gründe für den weiteren Verbleib des Strafvollzugs bei der Justiz geltend. Denn abschließend vertrat er wie bereits in einer vorangegangenen Besprechung mit Fischer und Oberst Kabanow - die Auffassung, die DVdI könne am besten gewährleisten, „daß der Strafvollzug im Sinne der Sicherung unserer demokratischen Ordnung durchgeführt wird“, und sprach sich dafür aus, „daß zur gegebenen Zeit der Strafvollzug als Ganzes der Deutschen Verwaltung des Innern eingegliedert wird“. „Aus politischen Gründen“ hielt er eine Übertragung zum damaligen Zeitpunkt jedoch für unzweckmäßig, da verhindert werden sollte, daß im Westen der Eindruck eines der justitiellen Kontrolle entzogenen Gefängniswesens entstand. Der DJV-Präsident, der noch im Juni 1949 ohne Wenn und Aber für die Beibehaltung des Strafvollzugs bei der Justiz eingetreten war, sah sich angesichts der Entschlossenheit seiner Kontrahenten in der Innenverwaltung, in der SED-Spitze und in der SMAD nicht länger in der Lage, seine Position rückhaltlos zu verteidigen, und verlegte sich daher aufs Taktieren. Daß der Sekretariatsbeschluß nicht umgesetzt wurde, konnte er als Erfolg seines Vorgehens im unmittelbaren Vorfeld der Staatsgründung der DDR verbuchen, als die führenden Politiker in der SBZ sich gegenüber dem Westen so wenige Blößen wie möglich geben wollten. Damit war die nach wie vor kontroverse Frage jedoch keineswegs endgültig entschieden. 


\section{Die schrittweise Übertragung des Strafvollzugs an das Innenministerium im Jahre 1950}

Als die SED-Führung im Zuge ihrer Beratungen in Moskau zur Vorbereitung der Gründung der DDR mit ihrem Schreiben vom 19. September 1949 unter anderem vorschlug, die Speziallager in der SBZ aufzulösen ${ }^{66}$, dachte sie dabei nicht an die Kompetenzerweiterung der deutschen Innenverwaltung; sie wollte vielmehr den zu gründenden ostdeutschen Staat nicht von vornherein mit einer zu schweren Hypothek belasten. Die sowjetische Seite erteilte am 27. September zwar ihre prinzipielle Zustimmung; während die SED-Führung jedoch empfohlen hatte, "die von den Sowjetorganen abgeurteilten Verbrecher nach der Sowjetunion zu transportieren und die übrigen den deutschen Organen zu übergeben“, wollte die Führung in Moskau aber einen Teil der Gefangenen entlassen und die verbliebenen den deutschen Behörden überstellen ${ }^{67}$. Nachdem am 28. September im Politbüro der KPdSU eine entprechende Entscheidung gefallen warb8, wurde in den ersten Oktobertagen der Innenverwaltung in Ost-Berlin „durch das Vertrauen der Besatzungsmacht die Durchführung des Strafvollzugs an den in den Lagern der Besatzungsmacht befindlichen Gefangenen übertragen" ${ }^{69}$. Ob die Übergabe der Gefangenen an die Justiz eine Alternative für Moskau darstellte, ist zweifelhaft. Denn die dem MWD unterstellten Lager waren für ehemalige Nationalsozialisten und andere Personen, insbesondere politische Gegner, bestimmt gewesen, die aus Sicht der Besatzungsmacht ein Sicherheitsrisiko darstellten. Der gleichsam ,natürliche' Ansprechpartner der sowjetischen Seite in dieser Frage war daher das Innenministerium.

Erste organisatorische Maßnahmen zur Übernahme des „gesamten Strafvollzug[s] der sowjetischen Militärverwaltung“ mit dem Ziel der Bildung einer Hauptabteilung Strafvollzug wurden am 31. Oktober 1949 in einer Besprechung beim neuen Chef der Deutschen Volkspolizei Kurt Fischer gefordert. Da jedoch über zentrale Fragen - wie etwa die Anzahl der Häftlinge und die Übernahme von Haftanstalten - noch keine Klarheit herrschte, konnten noch keine genauen Festlegungen getroffen werden 70 . Über genauere Informationen verfügte Staatssekretär Hans Warnke am 11. November, als er Chefinspekteur Gustav Röbelen, den künftigen Leiter der Hauptabteilung Haftsachen/Strafvollzug Karl Gertich, sowie Werner Gentz und seinen Mitarbeiter Karl Kroll zu einer weiteren Besprechung einlud. Warnke gab an, daß schätzungsweise 15-20000 Gefangene übernommen

66 Brief an Stalin, 19. 9. 1949, in: Badstübner/Loth, Pieck-Aufzeichnungen, S. 297; vgl. dazu Staritz, SED, Stalin und Gründung der DDR.

67 Die sowjetische Antwort geht indirekt aus den Vorschlägen des Politbüros an den Parteivorstand zur Bildung einer Provisorischen Regierung der DDR hervor: siehe Badstübner/Loth, Pieck-Aufzeichnungen, S. 305.

68 Gedruckt in: Sowjetische Speziallager in Deutschland, Bd. 2, S. 354 f. Siehe dort auch den Entwurf eines weiteren Politbürobeschlusses vom 19.10.1949, in dem erstmals das MdI als Ansprechpartner genannt wird (S. $355 \mathrm{f}$.).

69 Bericht der Hauptabteilung Strafvollzug, 10. 1. 1951, in: Buddrus, „im Allgemeinen“, S. 26.

70 Bericht vom 24. 11. 1949, BAB, DO1/11 Nr. 1508, Bl. 97-100; vgl. Eisert, Waldheimer Prozesse, S. $16 \mathrm{f}$. 
werden müßten, darunter ca. 2000 , die noch nicht verurteilt worden seien ${ }^{71}$. Da „die bestehenden Lager möglichst schnell zu liquidieren“ waren, mußten bisher der Justiz unterstellte Strafanstalten an das Innenministerium übergeben werden. Gentz kam zwar seinem Auftrag, Warnke eine Aufstellung über die Anzahl der Strafanstalten, ihre Fassungskapazität und ihre Belegungsfähigkeit zu übersenden, umgehend nach72. Das MdI verfügte jedoch erst nach weiteren vier Wochen über genauere Häftlingszahlen: Am 7. Dezember teilte Ulbricht Warnke mit, daß 10200 von Sowjetischen Militärtribunalen verurteilte sowie 3300 nicht verurteilte Gefangene übergeben und Buchenwald und Sachsenhausen als Haftlager aufgelöst würden ${ }^{73}$. Das einzige Lager, das das Innenministerium von der Besatzungsmacht übernehmen sollte, war Bautzen ${ }^{74}$. Nun stellte sich die Frage, welche Anstalten das Justizministerium zur Verfügung zu stellen hatte. Das MdI, dem die Angaben von Gentz nicht genügten, entschloß sich, selbst eine Inspektion der zehn größten Anstalten vorzunehmen. Als Gertich mit Gentz in dieser Angelegenheit Kontakt aufnahm, als Zweck der bevorstehenden Inspektionsreise aber lediglich angab, Informationen über den internen Betrieb der Anstalten zu erhalten, wurde dieser mißtrauisch und fragte, „ob seitens der Hauptverwaltung der Deutschen Volkspolizei die Absicht besteht, den gesamten Strafvollzug zu übernehmen". Fechner reagierte ähnlich wie Gentz und wiederholte gegenüber Gertich seine Auffassung, daß er den Zeitpunkt der Übernahme „für verfrüht halte“; außerdem hätte er „von dieser Frage die Übernahme des Justizministeriums mit abhängig gemacht"75. Erst auf seine Versicherung, „daß der Zweck der Informationen nichts mit dieser Angelegenheit zu tun" habe, erhielt Gertich schließlich die Ermächtigung zur Besichtigung der Haftanstalten.

Eine Übernahme des gesamten Strafvollzugs lag um die Jahreswende 1949/50 noch nicht in der Absicht des Innenministeriums. Daß es sehr viel weniger zimperlich mit den Gefangenen umzugehen beabsichtigte als die Justiz, war damals bereits klar. Ein MdI-Mitarbeiter plädierte dafür, die Häftlinge so zu beschäftigen, „daß sie sich nicht nur selbst erhalten, sondern auch die Verwaltungskosten durch nutzbringende Arbeit für die Gesellschaft abdecken können“. Und er fuhr fort: „Die bisherigen Methoden der Justiz können hier nicht automatisch übernommen werden, weil sie überaltet [sic] sind und nicht an das Ziel der Schaffung von brauchbaren und wertvollen Gliedern der Gesellschaft heranreichen. Es müssen deshalb vollkommen neue Wege beschritten werden. "Für das MdI stand die Ausbeutung der Arbeitskraft der Gefangenen im Mittelpunkt; daß Gefangenenarbeit ein Mittel zur Resozialisierung der Häftlinge darstellte, ging hingegen fast völlig

71 Diese Gefangenen, deren Anzahl sich auf 3442 belief, wurden in den sog. Waldheimer Prozessen verurteilt: siehe dazu u. a. Eisert, Waldheimer Prozesse; Werkentin, Scheinjustiz; Otto, Waldheimer Prozesse.

72 Vermerk Gertichs über die Besprechung im Innenministerium bei Warnke am 11.11. 1949, BAB, DO1/11, Nr. 1586; vgl. Eisert, Waldheimer Prozesse, S. 17-19; Buddrus, „im Allgemeinen“, S. 10.

73 Ebenda, S. 13.

74 Dies geschah am 15. 2. 1950: vgl. Finn, Politische Häftlinge, S. 162.

75 Vermerk über die Besprechung im Justizministerium zwecks Erteilung einer Ermächtigung zum Besuch bestimmter Strafanstalten, 20. 12. 1949, BAB, DO1/11, Nr. 1586, Bl. 21; vgl. Wunschik, Strafvollzug, S. 78. 
unter $^{76}$. Der Bericht über die Besichtigung der zehn größten Strafanstalten im Dezember zeigt zudem, daß die Bedürfnisse der Häftlinge den Interessen des Innenministeriums rücksichtlos untergeordnet wurden. $\mathrm{Da}$ die vom Justizministerium gemeldeten Belegungskapazitäten nicht ausreichend erschienen, „erfanden“ die MdI-Mitarbeiter die Kategorie der „höchstmöglichen Belegungsfähigkeit“: Konnten die zehn Anstalten nach den Vorstellungen der Justiz mit 8232 Personen belegt werden, so glaubte das MdI, 17200 Gefangene dort einpferchen zu können ${ }^{77}$.

Nach dieser Informationsreise legte Staatssekretär Warnke am 11. Januar von sich aus vier für seine $Z$ wecke geeignete Strafanstalten fest. Gentz wehrte sich jedoch gegen diese selbstherrliche Vorgehensweise, mit der das Justizministerium vor vollendete Tatsachen gestellt werden sollte, so daß nun über die Anstalten doch noch verhandelt werden mußte. Dabei gelang es den Vertretern des MdJ, die Übergabe der Strafanstalt Brandenburg-Görden zu verhindern; das Zuchthaus Waldheim mußten sie indes räumen, nicht zuletzt aufgrund rückhaltloser sowjetischer Unterstützung für das $\mathrm{MdI}^{78}$. Die Räumung der fünf Anstalten in Torgau, Luckau, Hoheneck, Untermaßfeld und Waldheim stellte die Strafvollzugsabteilung vor erhebliche Probleme: Zum einen wurde damit die Durchführung des Arbeitsplans stark beeinträchtigt ${ }^{79}$, und zum anderen bestand die Gefahr der Überbelegung der verbliebenen Anstalten. Da das Justizministerium dies vermeiden wollte, wurden auf Vorschlag von Gentz die Strafgefangenen, die zu nicht mehr als zwei Jahren Freiheitsstrafe verurteilt waren und keinen höheren Strafrest als fünf Monate hatten, ab Mai 1950 entlassen ${ }^{80}$; außerdem bemühte sich Fechner um die Freigabe weiterer, noch in sowjetischer Hand befindlicher Gefängnisse ${ }^{81}$. Für das MdI hatten sich die Ausgangsbedingungen in dem Kompetenzgerangel um den Strafvollzug entscheidend verbessert: Denn nun verfügte die Hauptverwaltung Deutsche Volkspolizei über sechs Haftanstalten mit insgesamt gut 14000 Häftlingen ${ }^{82}$, die seit dem 15. Februar ${ }^{83}$ von der Hauptabteilung Haftsachen/ Strafvollzug (HS) unter der Leitung von VP-Oberrat Karl Gertich verwaltet wurden ${ }^{84}$.

76 Bericht vom 24. 11. 1949, BAB, DO1/11 Nr. 1508, Bl. 99 f.

77 Vgl. Buddrus, „im Allgemeinen“, S. 14.

78 Vermerk Gertichs, 18.1. 1950, BAB, DO1/11, Nr. 1587, Bl.12f. Vgl. auch Eisert, Waldheimer Prozesse, S. 26f.; Buddrus, „im Allgemeinen“, S. 18, und Kobuch, Zur Rechtsprechung, S. 510 f., über die Probleme im Fall Waldheim.

79 So Gentz gegenüber Korobow am 15. 2. 1950, BAB, DP1 HAS III/145.

so Siehe Vermerk von Gentz für Fechner, 20. 1. 1950; Fechner an das Kleine Sekretariat und an Abt. Staatliche Verwaltung, 25. 1. 1950, BAB, DP1 VA Nr. 5808; Rundverfügung Nr. 59/50 vom 28. 4. 1950, BAB, DP1 VA Nr. 6414.

81 So etwa um die Freigabe des Landgerichtsgefängnisses in Dresden: siehe Fechner an Zentralsekretariat der SED, 13. 3. 1950, BAB, DP1 VA Nr. 262, Bl. 90.

82 Bericht der Hauptabteilung Strafvollzug, 10.1. 1951, in: Buddrus, „im Allgemeinen“, S. 26.

83 Datum nach dem Bericht der HVDVP für Kabanow, 26. 8. 1950, BAB, DO1/11, Nr. 1586, Bl. 4553 , hier 45.

84 Siehe Buddrus, „im Allgemeinen“, S. 15. Zu Gertich, der selbst im Dritten Reich aus politischen Gründen inhaftiert worden war, siehe Otto, Waldheimer Prozesse, S. 536, Anm. 17, und Fricke, Strafvollzug in Bautzen, S. 126. Er blieb nur bis Juli 1951 Hauptabteilungsleiter, als er aufgrund von Zugehörigkeit zu einer "trotzkistischen Gruppe“ verhaftet wurde. Im August 1952 zu sieben Jahren Zuchthaus verurteilt, wurde er 1956 entlassen. Anfang 1957 floh er nach West-Berlin. 
Daß die zwischen MdI und MdJ geteilte Zuständigkeit für den Strafvollzug keine Dauerlösung war, zeigte sich bereits im Frühjahr 1950. Denn zum 6. April wurde vom Leiter des SED-Justizsektors Berger eine Besprechung ,in Fragen des Strafvollzugs" mit den zuständigen Vertretern des Justiz- und des Innenministeriums anberaumt, in der es „zum allgemeinen Erstaunen“ der Teilnehmer um die kontroverse Frage der Unterstellung des Strafvollzugs gehen sollte. Die Besprechung war indes schlecht vorbereitet: Berger befand sich in Urlaub, und sein Vertreter Kampfrad erwies sich als uninformiert. Die Teilnehmer wollten außerdem vor einer Entscheidung ihre Minister hinzuziehen. Danach setzte sich Gertich mit Plenikowski in Verbindung, der die Besprechung absetzte, aber die Ausarbeitung einer Vorlage zu dieser Frage "durch das Referat Strafvollzug im ZS“"85 anordnete. Über das Politbüro sollte die Vorlage Warnke, Fischer und Fechner zugestellt werden, um diesen Gelegenheit zur Stellungnahme zu geben, damit dann „etwa notwendige Besprechungen durchgeführt werden können" ${ }^{86}$.

Fischer ergriff daraufhin die Initiative und versuchte, am 11. April durch eine Intervention bei Ulbricht eine Entscheidung in seinem Sinne herbeizuführen. In einer seinem Schreiben beigefügten Politbürovorlage verwies er auf die "gewaltige Überbelegung" der sechs Haftanstalten der Volkspolizei ${ }^{87}$, woraus sich für Fischer Sicherheitsprobleme und untragbare hygienische Verhältnisse ergaben. Wenngleich dies alles zutraf, so ist doch auffällig, daß nun nicht mehr von der „höchstmöglichen Belegungsfähigkeit" die Rede war; denn unter Zugrundelegung dieses Maßstabs hätte Fischer eine „gewaltige Überbelegung“ nicht geltend machen können. Seine Darlegungen bildeten somit nicht mehr als einen Vorwand, um die Strafvollzugsanstalten der Justiz zu erhalten, in denen angeblich „kein derartiger Raummangel“ bestehe ${ }^{88}$ und die sich zudem „durch vorzeitige Entlassung aller zu kurzen Freiheitsstrafen Verurteilten weiter bedeutend entlasten“ ließen. Des weiteren bezeichnete er den "nebeneinander bestehenden Strafvollzug" von Justiz und Polizei unter wirtschaftlichen und politischen Gesichtspunkten als unzweckmäßig und schlug dem Politbüro daher vor, zum 1. Juni 1950 den gesamten Strafvollzug unter der Leitung der HVDVP zu vereinigen ${ }^{89}$. Wie Fischer am folgenden Tag Steinhoff mitteilte, hatte er diesen Antrag „im Einvernehmen mit unseren Freunden", also mit Zustimmung der SKK-Innenverwaltung, gestellt ${ }^{90}$.

Fischers Vorlage bildete dann auch die Grundlage für die Beratung des SED-Sekretariats am 15. Mai 1950 über die „Vereinheitlichung des Strafvollzuges“. Das Sekretariat beschloß vorerst nur die Unterstellung des „Strafvollzug[s] an den nach Befehl 201 verurteilten Personen [...] mit allen Einrichtungen, mit ihren Stellenplänen und Haushaltsmitteln" unter die HVDVP91. Nach der Übergabe der Haftanstalten durch das MdJ im Januar/Februar bedeutete dies einen weiteren Schritt hin zu einem Polizeivollzug. Wie Paul Locherer, der Leiter der betroffenen

85 Ein solches Referat existierte nicht; es muß sich um ein Mißverständnis Gertichs handeln.

86 Aktenvermerk Gertichs betr. Besprechung im Zentralsekretariat am 5.4. 1950 [sic], BAB, DO1/ $11, \mathrm{Nr} .1586, \mathrm{Bl} .27 \mathrm{f}$.

87 Die auf 5834 Personen ausgelegten Anstalten waren Fischer zufolge mit 14621 Häftlingen belegt.

88 Dies traf, wie das MdJ am 17.5. 1950 der SKK mitteilte, nicht zu, BAB, DP1 HAS III/145.

89 Fischer an Ulbricht, 11. 4. 1950, Entwurf eines Politbürovorschlags, BAB, DO1/7 Nr. 49, Bl. $144 \mathrm{f}$.

90 Fischer an Steinhoff, 12. 4. 1950, ebenda, Bl. $147 \mathrm{f}$.

91 Protokoll der Sekretariatssitzung, 15. 5. 1950, TOP 2, SAPMO, DY 30 IV J 2/3/107. 
Strafanstalt Brandenburg-Görden, am 2. Juni Gertich berichtete, hatte Gentz ihm Ende Mai zwar mitgeteilt, daß die „201-Gefangenen“ nunmehr in den Strafvollzug der Volkspolizei übergehen müßten, daß aber „mit der Übergabe der Gefangenen noch nicht die Übergabe der Anstalt verbunden zu sein braucht“. Von Locherer darauf hingewiesen, "daß es falsch wäre, sich gegen die Entwicklung zu stellen, die im Zuge der Zeit läge“, und daß er „auf einem Ast sitze, der abgesägt werde“, entgegnete Gentz, „man müsse ausharren, bis die anderen beim Sägen müde würden“. Der Leiter der Strafvollzugsabteilung hoffte also, den Zug der Zeit zunächst noch aufhalten und dann in eine andere Richtung lenken zu können: Angesichts der verschlechterten Position des Justizministeriums in dem Kompetenzstreit mit dem Innenministerium und der nachgiebigen Haltung Fechners war dabei freilich der Wunsch Vater des Gedankens' ${ }^{92}$ Dies zeigte sich auch bei der Besprechung der Angelegenheit mit Fechner, der der Übergabe der „201er" einschließlich der Haftanstalten an die Polizei zustimmte ${ }^{93}$. In ihrem Bestreben, möglichst viel Haftraum, aber möglichst wenige Gefangene zu erhalten, wollte die HVDVP nur die „201er“ übernehmen, deren Strafrest noch mehr als vier Jahre betrug. Da dies jedoch eine weitere Belastung der bei der Justiz verbleibenden Strafanstalten bedeutet hätte, bestand Fechner am 7. Juni auf der buchstabengetreuen Ausführung des Sekretariatsbeschlusses ${ }^{94}$. Im Unterschied zu Gentz wollte er zwar den Sekretariatsbeschluß nicht unterlaufen; zu einem darüber hinausgehenden Entgegenkommen gegenüber der Polizei war jedoch auch er nicht bereit.

Noch vor Übergabe des Zuchthauses Brandenburg-Görden am 1. Juli 1950 erfolgte der nächste Schritt auf dem Weg zu einem Polizeivollzug. Als Anlaß bot sich die spektakuläre Flucht von zwei im Glauchauer Prozeß im Dezember 1948 verurteilten "Wirtschaftsverbrechern" aus dem Zuchthaus Zwickau mit Hilfe des Anstaltswerkmeisters und einer Justizwachtmeisterin. Auf der Sekretariatssitzung vom 12. Juni wurde der Bericht der "staatlichen Organe“ über diesen Vorgang - es handelte sich, wie ein ND-Artikel vom 18. Juni nahelegt, um einen ZKK-Bericht ${ }^{95}$ - zum Anlaß für den weitreichenden Beschluß genommen, „eine Kommission einzusetzen, mit der Aufgabe zu vereinbaren, daß der Strafvollzug in die Hände der Volkspolizei übergeht“. Bei der Besetzung der Kommission - mit Fechner (Vorsitzender), Polizeichef Fischer, Inspektor Röbelen und dem stellvertretenden Leiter der Abteilung Staatliche Verwaltung, Paul Hentschel - war die Justiz deutlich unterrepräsentiert ${ }^{96}$.

Angesichts dieser Entscheidung blieb Fechner nichts anderes übrig, als gute Miene zum bösen Spiel zu machen. Möglicherweise hoffte er, den Prozeß noch etwas hinauszögern zu können, wobei ihm Fischers Tod am 22. Juni zu Hilfe kam. Die Polizeiführung hielt freilich an dem Ziel, den Strafvollzug vollständig zu übernehmen, fest. Sorgfältig registrierte sie jede Flucht aus dem Justiz-Strafvoll-

92 Vgl. auch Wunschik, Strafvollzug, S. 78, der Gentz als „zweckoptimistisch“ bezeichnet.

93 Aktenvermerk Gertichs, 2. 6. 1950, BAB, DO1/11, Nr. 1586, Bl. 29.

94 Aktenvermerk Gertichs, 7.6. 1950, ebenda, Bl. 33.

95 Warum zwei Glauchau-Meeraner Wirtschaftsverbrecher flüchten konnten, in: Neues Deutschland, 18.6. 1950, S. 7. Der Artikel prangerte die Mißstände im Zuchthaus und den „humanen Strafvollzug“ an und forderte abschließend Garantien dafür, daß im Strafvollzug „streng nach den Gesetzen der Deutschen Demokratischen Republik verfahren wird“.

96 Protokoll der Sekretariatssitzung, 12. 6. 1950, SAPMO, DY 30 IV J 2/3/114, TOP 16. 
zug, um am 4. August Fechner das Ergebnis ihrer Beobachtungen mitzuteilen und darauf hinzuweisen, „daß die Strafvollzugs-Organe der Justiz ihre Aufgaben nicht mit genügendem Ernst durchführen, um Entweichungen von Strafgefangenen von vornherein auszuschalten "97. Aber Fechner hatte bereits vorher zu erkennen gegeben, daß er die Entwicklung nicht länger aufhalten wollte. Ausgehend von dem Zwickauer Ausbruchsfall referierte er am 17. Juli über „Probleme des Strafvollzugs“. Nachdem er Verdienste und Mängel des Gefängniswesens seit 1945 dargelegt hatte, erläuterte er abschließend, wie man die Flucht von Schwerverbrechern am besten verhindern könne: „Die ohne Zweifel unbefriedigende Bezahlung und sonstige materielle Lage der Wachmannschaften sind kein Ansporn. Es ist bekannt, daß vor 1918 und auch noch in der Weimarer Republik die großen Strafanstalten der Verwaltung des Innern unterstanden. Die heute über besser disziplinierte, besser ausgebildete und besser ausgerüstete Kader verfügende Verwaltung muß eingeschaltet werden. Und hier beginnt die Lösung des Problems. “98

Die Übertragung des Strafvollzugs auf das Innenministerium bedurfte freilich noch einer rechtlichen Grundlage. Die am 12. Juni 1950 eingesetzte Kommission kam zu dem Ergebnis, dies in einer Verordnung zu regeln"9, da bei einem Gesetzgebungsverfahren die Gefahr kontroverser Diskussionen mit den bürgerlichen Blockparteien in der Volkskammer oder in ihren Ausschüssen bestand, die man vermutlich vermeiden wollte. Mit der Ausarbeitung beauftragten Fechner und Warnke am 8. August Abteilungsleiter Nathan und Chefinspekteur Lust, die am selben Tag die Entwürfe einer entsprechenden Verordnung und von Durchführungsbestimmungen vorlegten ${ }^{100}$. Das Sekretariat stimmte am 16., das Politbüro am 22. August den gegenüber der Fassung vom 8. August nur geringfügig veränderten Entwürfen $z^{101}$. Damit billigten die Parteigremien eine Verordnung, die, wie Nathan am 8. August dargelegt hatte, der DDR-Verfassung widersprach. Denn Artikel 115 zufolge war die Ausführung von Republikgesetzen - also die Verwaltung - grundsätzlich Ländersache; dies galt bis zu diesem Zeitpunkt auch für den Strafvollzug. Nun sollte aber eine eigene Strafvollzugsverwaltung im Innenministerium errichtet werden. Da Artikel 115 für die Errichtung eigener Verwaltungen der Republik ausdrücklich ein Gesetz vorsah, war die Neuregelung des Gefängniswesens in einer Verordnung verfassungswidrig ${ }^{102}$. Mit dem Politbürobeschluß vom 22. August war die Entscheidung über die Übertragung des Strafvollzugs an das MdI endgültig gefallen. Der Regierungsbeschluß vom 16. November 1950 war demgegenüber nur noch eine Formalität, die an der Formulierung der Verordnung nichts änderte ${ }^{103}$. Da wesentliche Details der Übertragung jedoch

97 Seifert (Kommissarischer Chef der DVP) an Fechner, 4. 8. 1950, BAB, DP1 VA Nr. 5803.

98 Fechner, Probleme des Strafvollzuges. Kritik, Selbstkritik, praktische Lösung, 17. 7. 1950, BAB, DP1 VA Nr. 6997.

99 Vermerk Nathans, 8. 8. 1950, BAB, DP1 VA Nr. 7204, Bl. 20f. Darin schreibt er: „Dem Hauptentwurf ist entsprechend der erteilten Anweisung die Form einer Verordnung gegeben worden."

100 Ebenda, Bl. 22, $23 \mathrm{f}$.

101 Protokoll der Sekretariatssitzung, 16. 8. 1950, SAPMO, DY 30 IV J 2/3/131, TOP 2, Anlagen Nr. 1 und 2; Protokoll der Politbürositzung, 22. 8. 1950, SAPMO, DY 30 IV J 2/2/105, TOP 7, Anlagen Nr. 3 und 4. Als Datum der Übernahme wurde in beiden Beschlüssen der 1.11. 1950 genannt.

102 Vermerk Nathans, 8. 8. 1950, BAB, DP1 VA Nr. 7204, Bl. $20 \mathrm{f}$.

103 Protokoll der Regierungssitzung, 16. 11. 1950, BAB, DC 20 I/3 Nr. 36, Bl. 24; Gbl. 1950, S. 1165 f. 
im Herbst 1950 noch umstritten waren, konnte erst am 23. Dezember die erste Durchführungsbestimmung zu dieser Verordnung von MdJ und MdI erlassen werden ${ }^{104}$. Dabei stand die Frage, ob der HVDVP alle Haftanstalten oder lediglich die Vollzugseinrichtungen zu übergeben waren, im Mittelpunkt der Diskussion.

Die Polizei ging am 12. August noch davon aus, daß bei Übernahme des Strafvollzugs „sämtliche Untersuchungs- und Vollzugsanstalten einschließlich aller Haftlager, Haftkrankenhäuser und ähnlichen Anstalten in die unmittelbare Verwaltung der Hauptabteilung HS übergehen" ${ }^{105}$. Die SKK hielt es Mitte September jedoch für zweckmäßig, „wenn die HVDVP tatsächlich nur den Strafvollzug übernimmt, nicht aber auch noch die Verwaltung sämtlicher Untersuchungsanstalten" ${ }^{106}$. Wie deren Vertreter, Oberstleutnant Wlassow, später mitteilte, sah sie „die Übernahme der Vielzahl der kleinen Gerichtsgefängnisse als eine unnötige Belastung für die Volkspolizei an, da damit ein größerer Verwaltungsapparat verbunden wäre, der für die Häuser mit einem Fassungsvermögen von 15-100 Plätzen auch etatmäßig nicht tragbar wäre". Die SKK meldete sich somit erst nach den Entscheidungen der SED-Gremien zu Wort und betonte dabei, daß dies „keine Anweisung" sei, sondern die Entscheidung allein „bei den Organen der deutschen Regierung “ liege ${ }^{107}$. Wenngleich bei der HVDVP die Planungen für den Eventualfall der Übernahme aller Hafteinrichtungen weitergingen ${ }^{108}$, machten sich die Verantwortlichen in der Hauptabteilung HS die Auffassung der SKK zu eigen und schlugen nun vor, „nur die größeren Strafanstalten zu übernehmen und dort den Strafvollzug wahrzunehmen“, die Untersuchungshaftanstalten und Gerichtsgefängnisse aber weiterhin bei der Justiz zu belassen. Auf diese Weise hofften sie, die Einrichtung zusätzlicher Verwaltungsinstanzen für den Strafvollzug auf Länderebene vermeiden und die Kosten so gering wie möglich halten zu können ${ }^{109}$.

Dies kam Gentz entgegen, der auch nach dem Politbürobeschluß die Hoffnung nicht aufgab, zumindest einen Teil des Strafvollzugs nach seinen Vorstellungen durchführen zu können. Am 11. November verdeutlichte er Gertich, daß die Justiz für die Aufrechterhaltung der Untersuchungshaftanstalten eine Reihe von Kurzbestraften für Kalfaktorendienste benötige und zudem die bestehenden Haftlager aufgrund baulicher Mängel sehr schwer zu sichern und daher nur für Leichtbestrafte geeignet seien. „Der Sicherheitsfaktor für die Justiz“, so führte er in diesem Zusammenhang aus, „ist die Auswahl von kurzbestraften Gefangenen, die durch Familie oder Besitz in der Deutschen Demokratischen Republik so verankert sind, daß sie eine Flucht und die damit verbundene Fahndung nicht riskieren." Gentz’ Kalkül, daß die Polizei sich auf eine derartige „Sicherung“ nicht ein-

104 In: Ministerialblatt, 1950, S. $215 \mathrm{f}$.

105 Hauptabteilung HS an Seifert, 12. 8. 1950, BAB, DO1/11 Nr. 1586, Bl. $64 \mathrm{f}$.

106 Hausmitteilung HVDVP HS-Org. an Chef der DVP, 14. 9. 1950, BAB, DO1/11, Nr. 1586, Bl. 191. Die zitierte Äußerung stammte vom Leiter der SKK-Verwaltungsabteilung Kabanow.

107 Aktenvermerk Gertichs über eine Besprechung bei Wlassow am 12.10. 1950, BAB, DO1/11 Nr. 1577, Bl. 1 f.; vgl. Wunschik, Strafvollzug, S. 78 f.

108 Siehe den Vorschlag Gertichs vom 24. 10. 1950, BAB, DO1/11 Nr. 1586, BI. 99-104. Daß es sich um die Eventualplanung handelte, geht aus dem ersten Satz hervor.

${ }^{109}$ Entwurf betr.: Vorschlag zur Übernahme des Strafvollzuges in den Aufgabenbereich der HVDVP, 18. 10. 1950; Betr:: Übernahme des Strafvollzuges durch die Deutsche Volkspolizei, 9. 11. 1950, ebenda, Bl. 197-201, 113-116; das Zitat Bl. 114. 
lassen und vor den durch bauliche Maßnahmen oder personalintensive Bewachung entstehenden Kosten zurückschrecken würde, ging auf110. Am 16. November einigten sich Gentz und Gertich auf die Übernahme von lediglich neun Vollzugsanstalten und drei Haftkrankenhäusern durch die Volkspolizei, die in $\mathrm{Zu}$ kunft für die Verurteilten mit einer über zweijährigen Strafdauer sowie für die Strafgefangenen mit einem hohen Ausbruchsrisiko zuständig sein sollte. Die Justizverwaltung wollte hingegen die Untersuchungsgefangenen, die zu Jugendgefängnis Verurteilten und alle leichter bestraften, für einen gelockerten Strafvollzug in Haftlagern geeigneten Häftlinge in den ihr verbliebenen Einrichtungen unterbringen 111 .

Gentz handelte dabei freilich nicht im Einvernehmen mit Fechner, der sich nach den Entscheidungen vom August 1950 möglichst umgehend aller Haftanstalten und Häftlinge entledigen wollte ${ }^{112}$. Fechner konnte auch Ulbricht davon überzeugen, der Ende November dem Justizministerium mitteilte: „Die Hauptverwaltung Deutsche Vokspolizei soll alle der bisher dem Ministerium der Justiz unterstellten Vollzugsanstalten, Haftanstalten, Jugendanstalten und Haftlager übernehmen." Mit dieser am 30. November von Gentz verkündeten Entscheidung war die Absprache vom 16 . November hinfällig geworden ${ }^{113}$, was diesen interessanterweise nicht davon abhielt, bei der nächsten Zusammenkunft mit Vertretern der Hauptabteilung HS am 6. Dezember auch weiterhin auf der Grundlage der Vereinbarung vom 16 . November zu verhandeln ${ }^{114}$. Bei einer zwei Tage später angesetzten weiteren Besprechung, an der auch Fechner und Toeplitz teilnahmen, hielt sich Gentz indes an die Linie seines Ministers und verkündete, „daß der Ministerrat beschlossen habe, daß der gesamte Strafvollzug einschließlich aller Lager und Haftanstalten an die Hauptabteilung Volkspolizei [sic] übergehen soll“. Fechner bestand auf einem frühen Übernahmetermin, insbesondere bei den Untersuchungshaftanstalten ${ }^{115}$. Gegenüber Polizeichef Maron legte er später dar, warum er so auf das Tempo drückte: Zum einen sah der Strukturplan seines Ministeriums infolge des Regierungsbeschlusses vom 16. November ab Januar 1951 keine Strafvollzugsabteilung mehr vor, und zum anderen war das Aufsichtspersonal durch die bevorstehende Umstrukturierung in Unruhe versetzt worden, da es sich berechtigte Sorgen um seine Zukunft machte. Es kam zu Kündigungen, für die kein Ersatz beschafft werden konnte, und der Diensteifer der Strafvollzugsangstellten ließ nach, so daß die Sicherheit der Anstalten „erheblich beeinträchtigt" wurde116.

110 Vermerk Gertichs betr. Übernahme des Strafvollzuges durch die Deutsche Volkspolizei, 13. 11. 1950, ebenda, Bl. 125-133.

111 Niederschrift über die Besprechung zwischen Gertich und Gentz, 16. 11. 1950, ebenda, Bl. 150f.; vgl. Wunschik, Strafvollzug, S. 79.

112 Vgl. dazu Röbelen an Ulbricht, 24. 10. 1950, SAPMO, NY 4182/1096, Bl. 7.

113 Aktenvermerk Mehners über eine Besprechung im MdJ, 30.11. 1950, BAB, DO1/11 Nr. 1586, Bl. 181. Wie Wunschik, Strafvollzug, S. 79, in der Entscheidung Ulbrichts eine Bekräftigung der vorangegangenen Empfehlung der SKK sehen kann, ist unverständlich, hatte diese doch für eine Belassung der Untersuchungshäftlinge bei der Justiz plädiert.

114 Aktenvermerk Mehners über eine Besprechung im MdJ, 6. 12. 1950, BAB, DO1/11 Nr. 1586, Bl. 267.

115 Protokoll über die Sitzung im Justizministerium der DDR am 8. 12. 1950, 9. 12. 1950, ebenda, Bl. 277-280.

116 Fechner an Maron, 9. 12. 1950, ebenda, Bl. 271-273. Vorangegangen war ein Schreiben Fechners an das MdI vom 5. 12. 1950 (ebenda, Bl. 314), in dem er um die sofortige Übernahme von fünf Haft- 
Der Polizeichef legte die Verordnung keineswegs so weit aus wie Fechner, und machte dagegen geltend: „In diesem Beschluß ist weder von Untersuchungsanstalten die Rede, sondern nur vom Strafvollzug und Strafvollzugsanstalten, noch ist ein Termin genannt, bis zu welchem die Übergabe zu erfolgen hat." Außerdem wies er etwas süffisant darauf hin, daß es „nicht als Schuld der HVDVP bezeichnet werden" könne, wenn die Struktur des MdJ ab dem 1. Januar 1951 keine Strafvollzugsabteilung mehr vorsehe ${ }^{117}$. Maron blieb folglich bei der der Volkspolizei von der SKK nahegelegten, kostengünstigsten Option, nur die größeren Anstalten, nicht aber die Untersuchungshaftanstalten und Gerichtsgefängnisse zu übernehmen ${ }^{118}$.

Der Konflikt konnte nur dadurch gelöst werden, daß die Entscheidung, die Strafvollzugsabteilung im MdJ zum Jahresende 1950 aufzulösen, rückgängig gemacht wurde. Als Mitte Dezember, nach Verhandlungen mit dem Innenministerium, das Weiterbestehen der Hauptabteilung III im MdJ sichergestellt war ${ }^{119}$, erklärte sich auch Fechner „damit einverstanden, daß die Untersuchungshaftanstalten bis auf weiteres bei der Justizverwaltung bleiben“. Für die "Differenzen“ in der Auslegung des Regierungsbeschlusses machte der Staatssekretär im Justizministerium die Stellungnahme Ulbrichts verantwortlich, verschwieg dabei jedoch, daß es Fechner gewesen war, der diesen von seiner Auffassung überzeugt hatte ${ }^{120}$. Unter diesen Bedingungen einigten sich die beiden Ministerien, daß neben den Untersuchungshäftlingen alle Strafgefangenen mit einem Strafrest von weniger als neun Monaten beim MdJ verbleiben, während die übrigen Strafgefangenen dem MdI unterstellt werden sollten ${ }^{121}$. In der Durchführungsbestimmung vom 23. Dezember legten die beiden Ministerien daraufhin fest, daß zum 1. Januar 1951 die Verwaltung der Vollzugsanstalten Cottbus, Bützow-Dreibergen, Zwickau I, Bautzen, Coswig, Magdeburg-Sudenburg, Ichtershausen und Gräfentonna, der Haftkrankenhäuser Cottbus, Klein-Meusdorf und Schkeuditz sowie der Haftlager Rüdersdorf, Erlabrunn, Cranzahl-Sosa, Aue und Volkstedt auf das MdI übertragen wurde ${ }^{122}$. Im April 1951 einigten sich die beiden Ministerien auf die Übergabe weiterer acht Haftlager (Wittmannsdorf, Heidekrug, Pirna, Neschwitz, Bitterfeld, Krumpa, Pfännerhall, Maxhütte) und einer Vollzugsanstalt (Hohenleuben) in der Zeit vom 1. bis 15. Mai 1951'23. Trotz erheblicher Einbußen hatte Gentz vorerst noch die Fortexistenz seiner Abteilung, die ab 1951 die Bezeichnung "Justizhaftanstalten" trug, gesichert.

lagern gebeten hatte, und die ablehnende Antwort Marons vom 7. 12. 1950, BAB, DO1/11 Nr. 56, Bl. $1 \mathrm{f}$.

117 Maron an Fechner, 11. 12. 1950, ebenda, Bl. 3-6; vgl. Wunschik, Strafvollzug, S. $79 f$.

118 In der Frage der fünf yon Fechner genannten Haftlager zeigte er sich jedoch entgegenkommend und versprach, deren Übernahme „so sehr als möglich zu beschleunigen“ (ebenda, Bl. 3-6).

119 Siehe dazu Gentz an Maron, 14. 12. 1950, BAB, DO1/11 Nr. 1588, Bl. 291.

120 Toeplitz an Maron, 23.12. 1950, BAB, DO1/11 Nr. 1586, Bl. 315-317.

121 Dies geht aus dem Brief Marons an Steinhoff vom 6. 7. 1951, BAB, DO1/11 Nr. 1588, Bl. 173-175, hervor.

122 Erste Durchführungsbestimmung zur Verordnung zur Übertragung der Geschäfte des Strafvollzugs auf das Ministerium des Innern der Deutschen Demokratischen Republik, 23.12. 1950, in: Ministerialblatt 1950, S. 215 f.

123 Die Verhandlungen begannen am 18. 1. 1951: siehe die Vermerke vom 25. 1. 1951 sowie vom 20. 1. 1951, BAB, DO1/11 Nr. 1588, Bl. 153, 233-237. Die Einigung erfolgte auf einer Konferenz am 10. 4. 1951: siehe das Konferenzprotokoll vom 13. 4. 1951, ebenda, Bl. 218-222. 


\section{Die Arbeit der Hauptabteilung für das Anstaltswesen im MdJ bis zum Ende ihres Bestehens (1950-1952)}

Trotz aller Diskussionen um die Übernahme des Strafvollzugs durch die Volkspolizei setzte die Hauptabteilung III im MdJ ihre auf eine Strafvollzugsreform ausgerichtete Arbeit fort. Zur Förderung der Gefangenenarbeit wurde zum 1. März 1950 Paul Hussock eingestellt, der bis zum 1. Dezember 1950 als Hauptreferent in der Hauptabteilung Strafvollzug für das Referat „Arbeitsbeschaffung für Gefangene und Bewährungsarbeit für gestrauchelte Menschen“ zuständig war. Der ehemalige Leiter des Kreisarbeitsamtes Teltow war ein äußerst befähigter Fachmann auf diesem Gebiet ${ }^{124}$, dem es gelang, „binnen Jahresfrist mehrere Tausend Gefangene im Produktionsarbeitseinsatz unterzubringen"125. Insgesamt konnte im ersten Halbjahr 1950 der Arbeitseinsatz von Strafgefangenen bedeutend gesteigert werden. Leisteten 1949 nur etwa 30 Prozent der Gefangenen produktive Arbeit, so waren im zweiten Halbjahr 1950 „fast $70 \%$ in volkswirtschaftlich wichtigen Produktionszweigen tätig“. Dies war vornehmlich auf die Ausweitung der $\mathrm{Au}$ Benarbeit zurückzuführen; bei der Arbeitsbeschaffung für zu längerfristigen Strafen Verurteilte, die Arbeit innerhalb der Gefängnismauern benötigten, existierten nach wie vor Probleme ${ }^{126}$. Die Frage der Häftlingsarbeit kam auch auf einer gröBeren, von der Hauptabteilung Strafvollzug ins MdJ einberufenen Konferenz zur Sprache, die erneut verdeutlichte, daß Gentz die Gefangenenarbeit in erster Linie unter dem Gesichtspunkt der Resozialisierung sah. Er plädierte nicht nur für eine Ausweitung der Außenarbeit, sondern auch für die Verlagerung von Abteilungen volkseigener Betriebe in die Vollzugsanstalten und die Erteilung von Arbeitsaufträgen an die Gefängniswerkstätten ${ }^{127}$. Wenngleich der Vertreter des Ministeriums für Industrie sich wenig kooperativ zeigte ${ }^{128}$, war doch deutlich geworden, daß Gentz und seine Mitarbeiter auf diesem Gebiet eine beachtliche Aktivität entfalteten. Dazu zählte auch die Ausarbeitung einer Verordnung „über die Einplanung des Arbeitspotentials der Vollzugsanstalten“, die nach Absegnung durch das Sekretariat ${ }^{129}$ im April 1952 verabschiedet wurde. Sie knüpfte vor allem dadurch an die Strafvollzugsreform an, daß für jeweils zwei geleistete Arbeitstage drei Tage der Strafzeit als verbüßt galten; zudem konnte dem Strafgefangenen, der seine Arbeit „ständig bedeutend übererfüllt[e]“, ein Strafrest bis zu einem Jahr erlassen werden. Darüber hinaus regelte sie arbeitsrechtliche Fragen betreffend Entlohnung, Schutz der Arbeitskraft und Arbeitsruhe ${ }^{130}$.

124 Lebenslauf Hussock, 11. 2. 1950; Beurteilung Hussocks von Gentz, 24. 5. 1950, BAB, DP1 SE Nr. 74.

125 Beurteilung des Hauptreferenten Hussock von Gentz, 1. 2. 1951, ebenda.

126 Hussock, Arbeitseinsatz, S. 300 f. Zu den Zahlen vgl. auch Kern, Erziehung, S. 94. Seinen Aussagen zufolge stieg die Zahl der mit produktiver Arbeit beschäftigten Strafgefangenen Ende 1956 auf etwa 100 Prozent.

127 Niederschrift über die Arbeitsplanungskonferenz der Hauptabteilung Strafvollzug und Anstaltsverwaltung des MdJ am 9. 6. 1950, BAB, DP1 HAS III/114.

128 Vermerk über die Arbeitsbesprechung im MdJ am 9. 6. 1950, SAPMO, NY 4090/440, B1. $58 \mathrm{f}$.

129 Protokoll der Sekretariatssitzung, 28. 2. 1952, SAPMO, DY 30 J IV 2/3/272, TOP 11. Das Sekretariat folgte damit dem Justizbeschluß vom 11. 12. 1951, SAPMO, DY $30 \mathrm{~J}$ IV 2/2/182, TOP 6, Anlage 3, Punkt III.10.

130 Verordnung über die Beschäftigung von Gefangenen, 3. 4. 1952, GBl. 1952, S. 275 f. In der Begrün- 
Neben diesen späten Erfolgen bei der Arbeitsbeschaffung für die Häftlinge konnte die Hauptabteilung III Ende April 1951 die baldige Eröffnung einer „zentralen Ausbildungsstätte für Angestellte der Justizhaftanstalten“ in Naumburg ankündigen. Zunächst waren achttägige Kurzlehrgänge für alle Aufsichtsangestellten, ab 1952 Vierteljahreslehrgänge für Gefängnisleiter und neueingestelltes Personal vorgesehen ${ }^{131}$. Zwar bedeutete für Gentz die Eröffnung der Schule am 9. Juli $1951^{132}$ die Verwirklichung eines lange erstrebten Zieles, aber sie kam zu spät. Außerdem sollte der Unterricht das Aufsichtspersonal primär zur kompetenten Sicherung der Strafanstalten befähigen. Erst in zweiter Linie sollten den Hörern "gesellschaftliche Kenntnisse vermittelt werden, die sie befähigen, selbst zu erkennen, wie sie sich den einzelnen Gefangenen gegenüber zu verhalten haben und welche wichtige gesellschaftliche Funktion sie als Aufsichtsangestellte ausüben"133. Die jahrelangen Angriffe gegen die Justizverwaltung, sie vernachlässige die Sicherheit der Anstalten, hatte insofern Früchte getragen, als nunmehr das MdJ selbst, um keinen weiteren Kompetenzeinbußen Vorschub zu leisten, ebenfalls der Verhinderung von Entweichungen die erste Priorität zuschrieb ${ }^{134}$. Dennoch wurde, wie die Hauptabteilung Strafvollzug der HVDVP nach der Übernahme auch der Justizhaftanstalten negativ vermerkte, in der Naumburger Schulungsstätte „das Prinzip des psychologischen Eingehens auf die Persönlichkeit der einsitzenden Inhaftierten besonders hervorgehoben" ${ }^{135}$ und somit kein vollständiger Bruch mit den Ideen einer Strafvollzugsreform vollzogen.

Gentz, der 1951 im Zuge der Parteiüberprüfung in Bedrängnis geriet und fast aus der SED ausgeschlossen worden wäre ${ }^{136}$, hielt beharrlich an seinen Vorstellungen zur Strafvollzugsreform fest und geriet damit, auch im MdJ, immer mehr ins Abseits. In einer Beurteilung vom 20. September 1950 für die SKK hieß es: „Auf seinem speziellen Arbeitsgebiet des Strafvollzuges ist er zwar weltberühmt, jedoch sind seine sonst anerkannten Theorien in der gegenwärtigen Situation schwer zu verwenden. "137 Achtzehn Monate später bezeichnete der Leiter der Personalabteilung seine Arbeit zwar als „befriedigend“; einschränkend bemerkte

dung zur Verordnung (in: Protokoll der Regierungssitzung vom 3.4. 1952, BAB, DC 20 I/3 $\mathrm{Nr}$. 101, Bl. 68f.) wurde nicht auf die Vorarbeiten von Gentz eingegangen, sondern auf eine Vereinbarung vom Januar 1951 zwischen dem Minister für Schwerindustrie, dem MdJ und dem MdI über die Verwendung von Strafgefangenen als Bergarbeiter als Ursprung der Verordnung hingewiesen. Nunmehr habe der Chef der VP vorgeschlagen, „eine grundsätzliche Regelung zu treffen und auch andere Arbeiten einzubeziehen".

131 Rundverfügung Nr. 61/1951, 23. 4. 1951, BAB, DP1 VA Nr. 7311, Bl. 72-74.

132 Vgl. Ansprache des Ministers bei der Eröffnung der Schule für die Angestellten der Justizhaftanstalten der DDR am 9. 7. 1951, BAB, DP1 VA Nr. 6999.

133 Vgl. ebenda und Rundverfügung Nr. 61/1951, BAB, DP1 VA Nr. 7311, Bl. 72.

134 Dies geht auch daraus hervor, daß von Februar bis Mai 1951 nicht weniger als fünf Rundverfügungen des MdJ sich mit Gefangenenentweichungen befaßten: siehe Rundverfügung Nr. 23/1951, 5. 2. 1951; Nr. 57/1951, 21. 4. 1951; Nr. 58/51, 21. 4. 1951; Nr. 85/1951, 31. 5. 1951, alle in: BAB, DP1 VA Nr. 7311, Bl. 26, 68, 69, 107, sowie Rundverfügung Nr. 62/1951, 23. 5. 1951, BAB, DP1 VA Nr. 6284, Bl. 80.

135 Bericht über die Durchführung der Übernahme der Untersuchungshaftanstalten, Haftkrankenhäuser und Jugendhäuser der Justizverwaltung am 1.7. 1952, BAB, DO1/11 Nr. 1587, Bl. 162171 , hier 166.

136 Siehe Kap. B.II.3.

137 Beurteilung von Gentz, 20.9. 1950, in: BAB, DP1 VA Nr. 854, Bl. 10; in russischer Übersetzung ebenda, Bl. 4. 
er aber, daß Gentz „die Probleme des Strafvollzuges auf Grund unserer ökonomischen Verhältnisse nicht immer richtig zu lösen versteht" ${ }^{138}$. Gerade auf dem Gebiet, auf dem er jahrzehntelang Erfahrungen gesammelt und Anerkennung erworben hatte, wurde ihm nunmehr Inkompetenz vorgeworfen. Zwar konnte sich Gentz - wahrscheinlich mit Fechners Rückendeckung - noch bis Juni 1952 im MdJ halten; der Übergang auch der Justizhaftanstalten an die Polizei war jedoch nicht mehr zu verhindern ${ }^{139}$.

Hintergrund dieses letzten Aktes im Drama um die Verfügungsgewalt über die Hafteinrichtungen war der Mangel an Haftraum, der sich sowohl in den Justizhaftanstalten als auch in den Strafvollzugsanstalten bemerkbar machte. Aufgrund der stetig ansteigenden Belegung der Justizhaftanstalten mit Untersuchungsgefangenen - im März und April 1951 waren jeweils 500 neu eingeliefert worden wollte das MdJ die eigenen Anstalten möglichst entlasten. Fechner bat daher Innenminister Steinhoff am 15. Mai, ca. 4000 noch in den Justizhaftanstalten einsitzende Strafgefangene zu übernehmen. Denn zur Zeit säßen dort neben 7412 Untersuchungshäftlingen 5501 verurteilte Gefangene ein; zur „Aufrechterhaltung des technischen Dienstes“ in den Anstalten seien jedoch 1500 Strafgefangene ausreichend. Falls die Volkspolizei diese nicht übernehmen könne, solle sie sich einverstanden erklären, „daß die Justizverwaltung in Abweichung von der Regierungsverordnung vom 16.11.50 für die Ausnutzung dieser Arbeitskräfte wieder Strafvollzugslager errichtet“"140. Diesen Vorschlag wies der Innenminister umgehend zurück, hätte er doch die Aufgabe der alleinigen Zuständigkeit für die Strafvollzugseinrichtungen in der DDR bedeutet. Er bat Maron um eine Stellungnahme zu der Angelegenheit, insbesondere aber um eine Überprüfung, ,inwieweit noch Strafanstalten von der HVDVP übernommen werden können"141. Dessen Antwort war eine implizite Aufforderung, zur Lösung des Problems alle Strafanstalten zu übernehmen. Zum einen ging er davon aus, daß das MdJ bei der Vereinbarung vom Dezember 1950 unaufrichtig gewesen sei. Denn es verfüge, wie die Anfrage Fechners zeige, mit den Strafgefangenen mit einem Strafrest unter neun Monaten über weitaus mehr verurteilte Häftlinge, als für Kalfaktorendienste notwendig waren. Maron unterstellte daher dem MdJ, „daß von vornherein die Beibehaltung von Arbeitslagern zur Verstärkung der Haushaltsmittel der Justizverwaltung beabsichtigt war". Damit komme man aber "zu einem zweigeteilten Strafvollzug“, was einen Verstoß gegen die Verordnung vom 16. November 1950

138 Beurteilung von Gentz, 25. 4. 1952, ebenda, Bl. 2. Vgl. auch die Einschätzung zu Gentz im Bericht zur Überprüfung der Mitglieder und Kandidaten der SED vom Juli 1951, zit. bei Schöneburg, Freiheitsstrafen, S. 19: „Der Leiter, Gen. Gentz, ist ein bekannter Vertreter des humanistischen Strafvollzugs - und er hat nach diesen Erkenntnissen die HA personell aufgebaut. Er und seine Mitarbeiter gehen nicht vom Klassenstandpunkt heran. Sie können heute noch nicht verstehen, weshalb der Strafvollzug im Ministerium der Justiz der VP übertragen wurde, und haben deshalb für ihre Arbeit auch keine Konsequenzen gezogen."

139 Seine Bedenken („insbesondere politischer und justiztechnischer Art“) gegen die Übertragung trug Gentz am 14. 11. 1951 Major Schirajew von der SKK vor: siehe Vermerk Gentz, 15. 11. 1951, BAB, DP1 HAS III/145.

140 Fechner an Steinhoff, 15. 5. 1951, BAB, DO1/11 Nr. 1588, Bl. 186 f.; vgl. Wunschik, Strafvollzug, S. 81.

141 Thomas (persönlicher Referent Steinhoffs) an Chef der DVP, 24. 5. 1951, BAB, DO1/11 Nr. 1588, Bl. 183. 
bedeute. Zum anderen konstatierte er, daß die Haftanstalten des MdI in einem weit höheren Maße überbelegt seien als die des MdJ: Man verfüge über Haftraum für 17265 Gefangene, der Gefangenenstand belaufe sich aber auf 27192. „Diese Überbelegung“, so abschließend Maron, „wurde in sehr starkem Maße dadurch beeinflußt, daß von der HV Deutsche Volkspolizei wohl die Aufgaben des Strafvollzuges übernommen worden sind, aber nicht alle die Vollzugsanstalten, die für die Aufgaben des Strafvollzuges unter der Justizverwaltung verwandt wurden. Es konnte festgestellt werden, daß seitens der Justizverwaltung frühere Vollzugsanstalten als U-Haftanstalten benutzt werden." 142 Sowohl die Überbelegung der eigenen Anstalten als auch die Verhinderung eines "zweigeteilten Strafvollzugs“ legten eine Übernahme auch der Justizhaftanstalten durch die Volkspolizei nahe.

Die polizeiinterne Entscheidung darüber fiel wahrscheinlich in der ersten Augusthälfte. Denn am 23. August legte Volkspolizeikommandeur Jauch in einem Papier der Hauptabteilung Strafvollzug die "Richtpunkte“ nieder, „die bei der Übernahme der restlichen noch in den Händen der Justizverwaltung befindlichen Vollzugsanstalten und Gerichtsgefängnissen zu beachten sind“. Jauch gab zu bedenken, daß dies den Ausbau der Verwaltungsinstanzen für den Strafvollzug auf Länderebene bedeute ${ }^{143}$; dies wurde jedoch anscheinend ohne Widerspruch zur Kenntnis genommen. Darüber hinaus verdeutlichte er, daß die Hauptabteilung Strafvollzug im Gegensatz zu ihrer früheren Auffassung auch für die Übernahme des Jugendstrafvollzugs eintrete - ein weiterer Hinweis darauf, daß es keine geteilte Zuständigkeit für das Gefängniswesen mehr geben sollte ${ }^{144}$. Wenngleich auch außerhalb des MdI über die Übernahme der Justizhaftanstalten durch die Volkspolizei diskutiert wurde ${ }^{145}$, fiel eine Entscheidung erst im Rahmen des sogenannten "Justizbeschlusses“ des Politbüros am 11. Dezember 1951146. Am 20. Februar kamen schließlich ein Polizeivertreter und Gentz zu einer Besprechung "wegen der Übernahme der Justizhaftanstalten zum 1. Juli 1952“ zusammen $^{147}$. Die zweite Durchführungsbestimmung zur Verordnung vom 16. November 1950, die den Übergang der „Verwaltung aller Justizhaftanstalten, Justizjugendhäuser und Haftkrankenhäuser" mit Wirkung vom 1. Juli 1952 auf das MdI festlegte, wurde sogar erst am 5. Mai 1952 erlassen ${ }^{148}$. Dies bildete freilich nur noch die rechtliche Fixierung einer seit langem feststehenden Entscheidung.

142 Maron an Steinhoff, 6. 7. 1951, ebenda, Bl. 173-175; die Belegungszahlen in dem Konzept vom 23. 6. 1951 aus der Feder von VP-Kommandeur Jauch ebenda, Bl. 180f. Vgl. Wunschik, Strafvollzug, S. 81.

143 Zum 1. 1. 1951 waren bei den Landesbehörden der Volkspolizei Abteilungen Strafvollzug eingerichtet worden; da die damals übergebenen Einrichtungen jedoch zentral verwaltet werden konnten, fristeten diese bis zur Jahresmitte 1952 nur ein Schattendasein: siehe ebenda, S. 80 f.

144 Jauch, Richtpunkte, 23. 8. 1951, BAB, DO1/11 Nr. 1587, Bl. $63 \mathrm{f}$.

145 Noch am 14. 11. 1952 beklagte Gentz gegenüber Schirajew, daß dies „seit längerem erörtert, aber keine Entscheidung [...] getroffen wird“": siehe Vermerk Gentz, 15. 11. 1951, BAB, DP1 HAS III/ 145.

146 Protokoll der Politbürositzung, 11. 12. 1951, SAPMO, DY 30 J IV 2/2/182, TOP 6, Anlage 3, Punkt IV.10. Zum „Justizbeschluß" siehe Kap. B.IX.1.

147 Aktenvermerk betr. Übernahme der Justizhaftanstalten, 21.2. 1952, BAB, DO1/11 Nr. 1590, Bl. $321 \mathrm{f}$.

148 Ministerialblatt 1952, S. 47 f. 


\section{Der Strafvollzug als Polizeivollzug}

Nachdem die Volkspolizei sämtliche Haftanstalten übernommen hatte, stellte sie im Hinblick auf deren Sicherheit „erhebliche Mängel“ fest. Dies hing nicht nur mit dem immer noch deplorablen baulichen Zustand, sondern nach Auffassung eines Berichterstatters der Hauptabteilung Strafvollzug auch damit zusammen, daß „die Justizverwaltung der Sicherheit weit weniger Aufmerksamkeit gewidmet [hatte], als der ,Persönlichkeit" der Gefangenen“149. Dies sollte nun anders werden. Fortan kam der Sicherheit des Vollzugs absolute Priorität zu ${ }^{150}$, wenngleich gerade Anfang der fünfziger Jahre dieser Anspruch nicht immer eingelöst werden konnte. Denn auch die Polizei beklagte Gefangenenentweichungen, gegen die sie unter anderem mit entsprechenden Aufrufen und Artikeln in ihrer Zeitschrift „Die Volkspolizei“ vorzugehen suchte151. Aufgrund der neuen Prioritätensetzung strebte die Volkspolizei einen möglichst weitgehenden Austausch des Anstaltspersonals an, was sich jedoch nur schrittweise realisieren ließ. Bei der ersten Übernahmewelle Anfang 1951 gelang es zwar, die alten Anstaltsleiter "praktisch ohne Ausnahmen" durch neue zu ersetzen; zahlreiche Strafvollzugsangestellte auf der mittleren und unteren Ebene blieben vorerst freilich im Dienst. Erst im Laufe der Zeit wurden auch diese weitgehend ersetzt, so daß nur 5 Prozent des ursprünglichen Personals der 1950/51 übernommenen Anstalten im Vollzugsdienst verblieben. Etwas anders verhielt es sich 1952. Der Austausch der in den Justizhaftanstalten und Gerichtsgefängnissen beschäftigten Angestellten überstieg die Ressourcen der Volkspolizei, so daß bis auf 107 Personen zunächst alle übernommen wurden ${ }^{152}$.

Freilich mußten die aus der Justiz kommenden Mitarbeiter eine umfassende Schulung über sich ergehen lassen, und 1956 urteilte die Verwaltung Strafvollzug rückblickend: „Es war eine harte Arbeit, die Ideologie der aus der Justiz kommenden Mitarbeiter zu verändern." 153 Als völlig überholt galt dabei den neuen Herren im Strafvollzug die Auffassung, daß der Häftling ein gestrauchelter Mensch sei, der durch Erziehung wieder in die Gesellschaft integriert werden müsse. Nunmehr wurde eine völlig andere Einstellung des Wachpersonals gegenüber den Häftlingen gefordert, was Ende 1950 der neue Leiter des Zuchthauses Brandenburg-Görden, VP-Inspekteur Heinz Marquardt verdeutlichte: „Wir müssen lernen, die Gefangenen zu hassen. Wir wollen wieder aus diesem Haus ein Zuchthaus machen und kein Sanatorium. "154 Marquardt befand sich damit im Einklang mit dem auch für den Strafvollzug zuständigen SED-Funktionär, Anton Plenikowski, der auf einer Arbeitstagung am 3. Mai 1952 klarstellte, daß in der DDR

149 Bericht über die Durchführung der Übernahme der Untersuchungshaftanstalten, Haftkrankenhäuser und Jugendhäuser der Justizverwaltung am 1.7. 1952, BAB, DO1/11 Nr. 1587, Bl. 162171 , hier 166.

$150 \mathrm{Vgl}$. Oleschinski, Nur für den Dienstgebrauch, S. 10.

151 Vgl. u. a. Weber, Beseitigt die Mängel im Haftanstalts- und Gefangenentransportwesen, und Boje,

Gefangenenbewachung muß noch besser werden.

152 Zum Personalaustausch in den Strafanstalten vgl. Wunschik, Strafvollzug, S. $80 \mathrm{f}$.

153 Zit. ebenda, S. 80.

154 Zit. nach Finn, Politische Häftlinge, S. 170. 
„nicht arme bedauernswerte Menschen in Haft sitzen. Darum müsse mit der $\mathrm{Hu}$ manitätsduselei Schluß gemacht werden. "155

Unter dem Polizeiregime wurden daher wesentliche Errungenschaften der Strafvollzugsreform rückgängig gemacht. Daß Häftlinge „Blumenvasen, sonstige Galanteriewaren, eigene Schüsseln, Netze, Taschen usw.“ besaßen, hielten die Volkspolizisten für ebenso unnötig wie die Tätigkeit von Gefangenenräten und die Gewährung von Hafturlaub ${ }^{156}$. Hinzu kam, daß der Vollzug zu Beginn der fünfziger Jahre in zunehmendem Maße militarisiert und brutalisiert wurde. Der Volkspolizei erschien bei Übernahme der Anstalten „das Verhalten der Justizangestellten gegenüber ihren Vorgesetzten [...] lasch und durchaus nicht dazu angetan, erzieherisch auf die Gefangenen zu wirken“. Mangelnde Disziplin herrschte aus der Sicht des neuen Vollzugsorgans auch unter den Gefangenen, denen „das Erstatten von Meldungen über Belegungsstärke, das Erheben von Sitzplätzen beim Eintritt von Aufsichtskräften [...] größtenteils vollkommen unbekannt" gewesen sei ${ }^{157}$. Nun wurden wieder Umgangsformen in das Gefängniswesen eingeführt, die in ihrer militärischen Ausrichtung stark an die Zeit vor 1918 und vor 1945 erinnerten ${ }^{158}$. Daß der Vollzug unter der Ägide der Volkspolizei wieder brutaler wurde, registrierten selbst SKK-Mitglieder, die beobachtet hatten, wie ein alter Mann gefesselt durch die Straßen geführt worden war. Major Schirajew, der Gentz um Aufklärung über die einschlägigen Vorschriften bat, mißbilligte deutlich die „wahllose[.] Fesselung der Gefangenen“159. Derartige Maßnahmen widersprachen zwar den bisherigen Gepflogenheiten, ließen sich aber mit dem Verlangen nach größtmöglicher Sicherheit rechtfertigen. Dies traf zweifellos nicht auf die zunehmenden Schikanen in den Anstalten selber zu, wo Anfang der fünfziger Jahre oftmals aus nichtigen Anlässen Karzerstrafen erteilt und Häftlinge zum Teil schwer mißhandelt wurden ${ }^{160}$. Ein besonders grausames Regime herrschte anscheinend in der Vollzugsanstalt Torgau, wo 1950 „wie in keiner anderen DDRAnstalt der Gummiknüppel“ regierte161. Verwiesen sei in diesem Zusammenhang auch auf die rücksichtslose Niederschlagung der Unruhen vom 31. März 1950 in der Strafanstalt Bautzen, bei der Häftlinge Knochenbrüche, Gehirnerschütterungen und andere schwere Verletzungen davontrugen ${ }^{162}$.

Angesichts dieser Zustände war die Kontrolle des Strafvollzugs durch die Öffentlichkeit nicht länger erwünscht. 1948 hatte die SED eine völlig entgegengesetzte Auffassung vertreten und in ihrem weitreichenden Justizbeschluß vom Januar den „Aufbau der demokratischen Kontrolle des Strafvollzugs" durch den

155 Protokoll der Arbeitstagung am 3. 5. 1952 zur Vorbereitung der Übergabe der Justizhaftanstalten an das MdI, 6. 5. 1952, BAB, DP1 VA Nr. 295, Bl. 18-21, hier 20.

156 Wunschik, Strafvollzug, S. 81; Bericht über die Durchführung der Übernahme der Untersuchungshaftanstalten, Haftkrankenhäuser und Jugendhäuser der Justizverwaltung am 1.7. 1952, BAB, DO1/11 Nr. 1587, Bl. 169.

157 Ebenda, Bl. 168.

158 Oleschinski, Strafvollzug in Deutschland, S. 65. Zur zunehmenden Militarisierung des Strafvollzugs siehe auch die Erlebnisberichte der Bautzen-Häftlinge, in: Gelbes Elend, S. 132f., 166, 172.

159 Vermerk von Gentz über die Rücksprache in Karlshorst am 14.11. 1951 mit Schirajew, 15. 11. 1951, BAB, DP1 HAS III/145.

160 Vgl. Finn, Politischer Strafvollzug, S. 108.

161 Finn, Hinter dem Eisernen Vorhang, S. 218.

162 Siehe dazu Fricke, Strafvollzug in Bautzen, S. 134-136. 
Strafvollzugsausschuß des Landtags und Ausschüsse an den Orten der Strafanstalten als "wichtige rechtspolitische Aufgabe" bezeichnet ${ }^{163}$. Dementsprechend wurde sogar das Plädoyer Erich Zeigners im sächsischen Landtag zur Bildung eines Strafvollzugsausschusses in der "Neuen Justiz" abgedruckt ${ }^{164}$. Zwei Vorgänge aus den Jahren 1951/52 verdeutlichen, daß die Staats- und Parteiführung nach dem Übergang des Strafvollzugs an das Innenministerium diese Ausschüsse nicht mehr förderte, sondern vielmehr massiv behinderte und sogar auflösen wollte.

Anfang Juli 1951 hatte eine Abordnung des Kreisstrafvollzugsausschusses Bautzen die seit dem 1. Januar der Volkspolizei unterstellte Haftanstalt Bautzen II 165 besichtigt und festgestellt, welche haarsträubenden Verhältnisse - vor allem im Hinblick auf Ernährung und Hygiene - dort herrschten. Ein von einem LDPVertreter angefertigter Bericht ${ }^{166}$ gelangte über die Vorsitzende des Strafvollzugsausschusses im sächsischen Landtag, Gertrud Thürmer (ebenfalls LDP) und Landtagspräsident Otto Buchwitz an Ministerpräsident Grotewohl167. Mit der Überprüfung der Haftanstalt beauftragte dieser die Hauptabteilung Strafvollzug bei der HVDVP, deren Untersuchungskommission erwartungsgemäß feststellte, daß der Bericht vom Juli 1951 nicht den Tatsachen entsprach ${ }^{168}$. Grotewohl gab sich damit zufrieden und übersandte das gesamte Material an Buchwitz mit einem geharnischten Begleitbrief. Darin hob er nicht nur hervor, „in welch verantwortungsloser Weise Kreise der LDP sich hier zum Fürsprecher unserer politischen Feinde gemacht haben“, sondern auch, "welche unverantwortlichen Wirkungen aus der Arbeit solcher Ausschüsse entstehen können“. Er könne sich daher dem Vorschlag von Staatssekretär Warnke "nicht verschließen, daß es ratsam ist, die Tätigkeit solcher Kontrollausschüsse für den Strafvollzug sofort einzustellen"169. Damit war die Sache erledigt, und Buchwitz blieb nur noch, das Ergebnis der Überprüfung dem Kreisstrafvollzugsausschuß am 25. Februar 1952 mitzuteilen; die Empfehlung, daß die Strafvollzugsausschüsse ihre Tätigkeit einstellen sollten, gab er jedoch nicht weiter 170 .

Der zweite Vorstoß in diesem Zusammenhang kam ebenfalls aus Sachsen. Der Strafvollzugsausschuß des sächsischen Landtags wandte sich im Januar 1951 angesichts der Zentralisierung des Strafvollzugs durch die Verordnung vom 16. November 1950 an den Landtagspräsidenten mit der Bitte, der Volkskammer vorzuschlagen, dort ebenfalls einen Strafvollzugsausschuß ins Leben zu rufen. Buchwitz leitete am 29. Januar den Antrag des Strafvollzugsausschusses an Volkskam-

163 Gedruckt in: Amos, Justizverwaltung, S. 232; vgl. auch Chef der DJV an Landesregierungen/Justizministerium, 27. 12. 1948, BAB, DP1 VA Nr. 839, Bl. 42-44, hier $43 \mathrm{f}$.

${ }^{164} \mathrm{Vgl}$. Zeigner, Bildung. Der Antrag wurde am 10. 12. 1948 eingereicht und wenig später verabschiedet: ebenda, Anm. 1.

165 Nicht zu verwechseln mit der Strafvollzugsanstalt Bautzen I, die im Februar 1950 von der Besatzungsmacht der Volkspolizei übergeben wurde.

166 E. Weber, Bautzen, an LDP-Landesvorstand Sachsen, z.H. Frau Thürmer, 13. 7. 1951, SAPMO, NY 4090/440, Bl. $113 \mathrm{f}$. Der Schlußsatz des Schreibens lautete: „Aus diesem Bericht ist zu ersehen, wie nötig es ist, daß dem Kontrollausschuß auch die der Volkspolizei unterstehenden Haftanstalten zugänglich gemacht werden."

167 Buchwitz an Grotewohl, 5. 9. 1951, ebenda, Bl. 112.

168 Stellungnahme der Hauptabteilung Strafvollzug zum Schreiben des Präsidenten des Sächsischen Landtags vom 5. 9. 1951, ebenda, Bl. 93-97.

:69 Grotewohl an Buchwitz, o.D. [Oktober 1951], ebenda, Bl. 109.

170 Siehe Buchwitz an Grotewohl, 17.3. 1952, ebenda, Bl. 92. 
merpräsident Dieckmann weiter ${ }^{171}$. Am 9. März diskutierte das Präsidium der Volkskammer über den Antrag, und der „streitbare[.] und häufig eigensinnige[.]“ Dieckmann ${ }^{172}$, der die Notwendigkeit „eines demokratischen Strafvollzuges“ mehrfach betonte, unterstützte das Vorhaben ${ }^{173}$. Ein der SED angehörender Sitzungsteilnehmer vermutete wohl zu Recht einen Zusammenhang mit einem vergeblichen Versuch Dieckmanns vom 27. Februar, die Kompetenzen des Gnadenausschusses der Volkskammer auf "allgemeine Fragen der Gnadenausschüsse“ auszuweiten und Kontakte zu den Gnaden- und Strafvollstreckungsausschüssen der Landtage aufzunehmen ${ }^{174}$. Auch Sekretariat und Politbüro befaßten sich am 2. und 3. April mit dem LDP-Vorstoß, lehnten die Bildung eines Strafvollzugsausschusses aber ab ${ }^{175}$. Damit war auch die Entscheidung des Ministerrats präjudiziert, der in seiner Sitzung am 23. August 1951 den Antrag formell zurückwies ${ }^{176}$. Angesichts des Bedürfnisses der Staatsführung, die Verhältnisse im Strafvollzug den Augen der Öffentlichkeit zu entziehen, hatte eine derartige Initiative daher keine Chance.

Die Strafvollzugsausschüsse der Landtage mußten dennoch nicht verboten werden, da die föderalen Parlamente nur noch bis zur Auflösung der Länder im Sommer 1952 existierten. Als Kontrollorgan für den Strafvollzug fungierte zudem seit dem 27. März 1952, dem sowjetischen Vorbild entsprechend, der Generalstaatsanwalt, dem mit einem Ministerratsbeschluß unter anderem „die Aufsicht über alle Haft- und Strafvollzugsanstalten übertragen" wurde ${ }^{177}$. Der Konkretisierung diente eine Rundverfügung vom 31. März, die am 29. Februar Gegenstand einer Aussprache zwischen einem Vertreter der Obersten Staatsanwaltschaft und zwei VP-Offizieren gewesen war. In ihrem Bestreben, die Eingriffsrechte der Staatsanwälte möglichst eng zu begrenzen, wandten sich letztere gegen einen Passus des Entwurfs, demzufolge der kontrollierende Staatsanwalt die Abstellung festgestellter Mängel zu überwachen habe, da dies eine unmittelbare Anordnungsbefugnis des Staatsanwalts in den Gefängnissen impliziere. Außerdem wollte die Volkspolizei die der HVDVP unmittelbar unterstehenden Anstalten - in der die politischen Gefangenen einsaßen - von der Kontrollbefugnis der Staatsanwaltschaft ausschließen ${ }^{178}$. Die Volkspolizei konnte sich zwar mit ihrem ersten, nicht

171 Buchwitz an Dieckmann, 29. 1. 1951, BAB, DC 20 I/3 Nr. 65, Bl. 125.

172 So die zutreffende Charakterisierung von Sommer, Liberal-Demokratische Partei Deutschlands, S. 158.

173 Vermerk über die Sitzung des Präsidiums der Volkskammer vom 3.3. 1951, BAB, DC 20 1/3 Nr. 65, Bl. 126.

174 Vermerk über die Sitzung der Gnadenausschusses der Volkskammer vom 27. 2. 1951, ebenda, Bl. 127; Niederschrift über die 2. Sitzung des Gnadenausschusses am 27. 2. 1951, ebenda, Bl. 128131. Hintergrund dieser Initiative bildeten wohl Dieckmanns Erfahrungen mit den Waldheimer Prozessen. Gemeinsam mit seinem Parteifreund und Schwager Walter Thürmer hatte er sich im sächsischen Gesamtministerium am 31.8. 1950 der Aufforderung des Berliner Justizministeriums widersetzt, die Gnadengesuche der in Waldheim zum Tode Verurteilten abzulehnen: siehe Kobuch, Zur Rechtsprechung, S. $518 \mathrm{f}$.

175 Protokolle der Sekretariatssitzung, 2. 4. 1951, und der Politbürositzung, 3. 4. 1951, SAPMO, DY $30 \mathrm{~J}$ IV $2 / 3 / 183$, TOP 57 , und DY $30 \mathrm{~J}$ IV $2 / 2 / 141$, TOP 6.

176 Protokoll der Regierungssitzung, 23. 8. 1951, BAB, DC 20 I/3 Nr. 65, Bl. 120. Bei gleicher Gelegenheit wurde auch eine Ausweitung der Kompetenzen des Gnadenausschusses abgelehnt.

177 Gedruckt in: Ministerialblatt 1952, S. 35. Vgl. Kap.B.VII.6.

178 Maron an Melsheimer, 5. 3. 1952, BAB, DO1/11 Nr. 1589, Bl. $46 f$. 
aber mit dem zweiten Änderungsvorschlag durchsetzen ${ }^{179}$. Daß diese Kontrollen sich auf die Haftbedingungen positiv auswirkten, erscheint jedoch zweifelhaft. Ein Bericht der Obersten Staatsanwaltschaft aus dem zweiten Halbjahr 1954, demzufolge „der überprüfende Staatsanwalt [...] für die U[ntersuchungs]- und Strafgefangenen schon der anerkannte Helfer geworden" sei, so daß die Häftlinge bei dessen periodischen Besuchen um eine Vorführung bäten ${ }^{180}$, klingt nicht nur äußerst schönfärberisch; er widerspricht auch dem Befund, daß „die jahrelang beobachteten schweren Verletzungen elementarer Bestimmungen im Strafvollzug" nicht verhindert wurden und daß „es politischen Häftlingen in der Praxis häufig überhaupt verwehrt wurde, zum aufsichtführenden Staatsanwalt vorzudringen oder schriftliche Beschwerden an ihn zu richten"181. Die Kontrolle durch ein parlamentarisches Gremium war eben nicht durch die eines Staatsorgans zu ersetzen, das sich primär für die Effizienz des Strafvollzugs, aber kaum für die Achtung der Menschenrechte der Gefangenen interessierte.

Nachdem seit 1948 der Verbleib der Haftanstalten bei der Justiz in Frage gestellt worden war, gab es immer wieder Vorstöße der Innenverwaltung, diese Kompetenz an sich zu ziehen. Vorerst konnte Fechner sich jedoch diesen Bestrebungen - nicht zuletzt unter Verweis auf den fatalen Eindruck, den ein solcher Schritt im Westen erwecken würde - erfolgreich widersetzen. Mit der 1950 im wesentlichen erfolgten Übertragung des Strafvollzugs auf das MdI war zwar eine Angleichung an die Kompetenzverteilung in der Sowjetunion erfolgt; es handelte sich dabei jedoch keineswegs um die simple Ausführung einer sowjetischen Anordnung, sondern um einen komplexen Entscheidungsprozeß, an dem deutsche Stellen anscheinend in einem höheren Maße beteiligt waren als sowjetische. Die Sowjetunion hatte mit der Übergabe eines Teils der Insassen der Speziallager an die Volkspolizei zwar die Ausgangsposition des MdI in der Auseinandersetzung um den Strafvollzug gegenüber dem MdJ gestärkt; die Initiative, die letztlich zum Erfolg führte, ging hingegen von Polizeichef Fischer aus, der sich freilich mit den "Freunden" abstimmte. Der Empfehlung der SKK an die HVDVP vom Herbst 1950, lediglich die größeren Anstalten zu übernehmen, kam keineswegs die Bedeutung zu, die ihr jüngst attestiert worden ist ${ }^{182}$. Entscheidend war vielmehr die enge Koalition zwischen Ulbricht, dem SED-Apparat und der Leitung der Volkspolizei, gegen die das MdJ allein nicht ankam. Auch daß zum Jahresende nicht das gesamte Gefängniswesen, sondern nur die Strafvollzugsanstalten, einige Haftlager sowie die Haftkrankenhäuser in die Verfügungsgewalt des MdI übergingen, läßt sich nicht primär auf den sowjetischen Ratschlag, sondern auf das Gewicht dieser machtvollen Verbindung zurückführen. Solange Ulbricht für eine sofortige Übertragung des gesamten Strafvollzugs eintrat - und damit gemeinsam mit Fechner den Vorstellungen der Polizeiführung widersprach - war diese Koalition hand-

\footnotetext{
179 Siehe Rundverfügung Nr. 10/52, 31. 3. 1952, BAB, DP3 III Nr. 121.

180 Die Aufsicht über die Haftanstalten, o.D., o.V. [Oberste Staatsanwaltschaft, 2. Jahreshälfte 1954], ebenda.

181 Finn, Politischer Strafvollzug, S. 28.

182 Wunschik, Abteilung Strafvollzug, S. 78, führt die Verordnung vom 16. 11. 1950 auf diese Empfehlung zurück, berücksichtigt dabei jedoch nicht die vorangegangenen Beschlüsse des Sekretariats und des Politbüros, die ohne erkennbare sowjetische Einflußnahme gefaßt worden waren.
} 
lungsunfähig. Daß die Konfrontation zwischen Fechner und Maron schließlich im Sinne der Volkspolizei gelöst wurde, verweist darauf, daß das „bewährte“ Bündnis wiederhergestellt worden war.

Die Übertragung des Gefängniswesens auf das Innenministerium hatte weitreichende Folgen für die Ausgestaltung des Strafvollzugs. Dies kündigte sich bereits 1948/49 an, als die Justizverwaltung unter dem Druck der Innenverwaltung mehr und mehr die Sicherheit der Anstalten in den Vordergrund rückte und der Humanisierung des Strafvollzugs demgegenüber einen deutlich geringeren Stellenwert zusprach. Solange die Haftanstalten jedoch der Justiz unterstellt blieben, konnte dort ein Mindeststandard menschenwürdiger Verhältnisse aufrechterhalten werden, nicht zuletzt dank Werner Gentz, der bis zu seinem Ausscheiden aus dem MdJ an der Verwirklichung seiner Reformideen arbeitete. Mit dem Übergang des Strafvollzugs an das Innenministerium, das nicht mehr die Resozialisierung der Gefangenen, sondern deren sichere Verwahrung und eine möglichst optimale Ausnutzung von deren Arbeitskraft in den Mittelpunkt seiner Bemühungen stellte, wurden diese Reformbestrebungen jedoch endgültig zunichte gemacht. 